\title{
Top and beauty synergies in SMEFT-fits at present and future colliders
}

\author{
Stefan Bißmann, Cornelius Grunwald, Gudrun Hiller and Kevin Kröninger \\ Fakultät Physik, TU Dortmund, \\ Otto-Hahn-Str. 4, Dortmund D-44221, Germany \\ E-mail: stefan.bissmann@tu-dortmund.de, \\ cornelius.grunwald@tu-dortmund.de, ghiller@physik.uni-dortmund.de, \\ kevin.kroeninger@tu-dortmund.de
}

ABSTRACT: We perform global fits within Standard Model Effective Field Theory (SMEFT) combining top-quark pair production processes and decay with $b \rightarrow s$ flavor changing neutral current transitions and $Z \rightarrow b \bar{b}$ in three stages: using existing data from the LHC and $B$-factories, using projections for the HL-LHC and Belle II, and studying the additional new physics impact from a future lepton collider. The latter is ideally suited to directly probe $\ell^{+} \ell^{-} \rightarrow t \bar{t}$ transitions. We observe powerful synergies in combining both top and beauty observables as flat directions are removed and more operators can be probed. We find that a future lepton collider significantly enhances this interplay and qualitatively improves global SMEFT fits.

Keywords: Beyond Standard Model, Effective Field Theories

ArXIV EPRINT: 2012.10456 


\section{Contents}

1 Introduction $\quad 2$

2 Effective theory setup 3

2.1 SMEFT dimension-six operators 3

2.2 Flavor and mass basis 4

2.3 Matching and running: SMEFT and WET 6

2.3.1 SMEFT RGE 6

2.3.2 Matching SMEFT onto WET $\quad 7$

2.3.3 WET RGE 8

3 Observables $\quad 8$

3.1 Top-quark observables $\quad 8$

$3.2 Z b \bar{b}$ observables $\quad 9$

$3.3 \quad B$-physics observables $\quad 9$

$\begin{array}{ll}3.4 & \text { Sensitivity to BSM contributions }\end{array}$

4 Fits to present data $\quad \mathbf{1 2}$

4.1 Current constraints from top-quark measurements at the LHC 13

$\begin{array}{lll}4.2 & \text { Constraints from } Z b b \text { measurements } & 14\end{array}$

$\begin{array}{lll}\text { 4.3 Current constraints from } B \text { physics measurements } & 14\end{array}$

$\begin{array}{lll}4.4 & \text { Combined fit to current data } & 17\end{array}$

5 Impact of future colliders $\quad 19$

5.1 Expected constraints from HL-LHC and Belle II 19

$\begin{array}{lll}5.2 & \text { CLIC projections } & 21\end{array}$

5.3 Combined fit 22

6 Conclusions $\quad 24$

$\begin{array}{ll}\text { A Weak effective theory } & 26\end{array}$

$\begin{array}{ll}\text { B SMEFT coefficients in the mass basis } & 27\end{array}$

$\begin{array}{ll}\text { C SMEFT operators in the mass basis } & 27\end{array}$

D Analytic formulas for one-loop matching 28

$\begin{array}{ll}\text { E Numerical matching conditions } & 31\end{array}$

F $\quad$ Auxiliary plots $\quad 32$ 


\section{Introduction}

Physics beyond the Standard Model (BSM) has and is being intensively searched for at the Large Hadron Collider (LHC) and predecessor machines. However, despite the large amount of data analyzed, no direct detection of BSM particles has been reported to date. Thus, BSM physics could be feebly interacting only, has signatures not covered by the standard searches, or is simply sufficiently separated from the electroweak scale. The latter scenario opens up a complementary approach to hunt for BSM physics at high energy colliders, in a similar spirit as the high luminosity flavor physics programs in pursuit of the precision frontiers with indirect searches. In this regard, the Standard Model Effective Field Theory (SMEFT) offers both a systematic and model-independent way to parametrize BSM contributions in terms of higher-dimensional operators constructed out of Standard Model (SM) fields and consistent with SM symmetries. At energies below the scale of BSM physics, $\Lambda$, this framework allows to perform global fits which could give hints for signatures of BSM physics in different observables and sectors simultaneously.

In recent years, this approach played a crucial role in the top-quark sector of SMEFT [1-17]. The SMEFT framework also allows the combination of top-quark data with $B$ data [18-23], which, thanks to different sensitivities, significantly improves constraints on SMEFT coefficients [18].

In this work, we extend previous works and analyze sensitivities to semileptonic fourfermion operators. The reason for doing so goes way beyond of making the fit more modelindependent: firstly, semileptonic four-fermion operators are presently of high interest as they are the agents of the flavor anomalies, hints of a breakdown of the SM in semileptonic $b \rightarrow s$ decay data [24]. Secondly, these operators provide contact interactions of top quarks and leptons, which could be studied ideally at future lepton colliders, e.g. ILC [25, 26], CLIC [27] or FCC [28], as discussed in refs. [29-41]. We intend to specifically work out and detail the interplay of constraints for operators with gauge bosons, that is, covariant derivatives in the SMEFT language, and four fermion operators in top-pair production processes, $Z \rightarrow b \bar{b}$ and $b \rightarrow s$ transitions for three stages: today, combining existing LHC, $Z b b$ and $B$-factory data, near future, adding projections from HL-LHC [42] and Belle II [43], and the far future, putting all together with lepton collider input, for the concrete example of CLIC [27]; we investigate how a future lepton collider impacts constraints and opens up new directions for testing BSM physics precisely.

This work is organized as follows: in section 2 we introduce the dimension-six SMEFT operators considered in this work and the low-energy effective field theories (EFTs) employed to compute SM and BSM contributions to $B$ observables. We also present the matching between SMEFT and weak effective theory (WET) and highlight how SU(2) $L$ invariance of the SMEFT Lagrangian allows to relate top-quark physics and $b \rightarrow s$ flavorchanging neutral currents (FCNCs). In section 3 we discuss the sensitivity of different observables to the various effective operators considered. Fits to present top-quark, $Z b b$, and $B$ data are presented in section 4 . We analyze how the complementary sensitivity of the observables from top-quark, $Z b b$, and $B$ sectors improves constraints on the SMEFT coefficients. In section 5 we consider different future scenarios, and detail on the ques- 
tion how measurements at a future lepton collider can provide additional information on SMEFT coefficients. In section 6 we conclude. Additional information is provided in several appendices.

\section{Effective theory setup}

In this section we give the requisite EFT setup to describe BSM contributions to topquark and beauty observables. We introduce the SMEFT Lagrangian in section 2.1, and identify the effective operators contributing to interactions of third-generation quarks. Consequences for FCNCs that arise from flavor mixing are worked out in section 2.2, where we also highlight the complementarity between contributions from up-type and down-type quarks. The matching conditions for $B$ observables in the low energy effective Lagrangian in terms of SMEFT coefficients are detailed in section 2.3.

\subsection{SMEFT dimension-six operators}

At energies sufficiently below the scale of new physics, $\Lambda$, the effects of new interactions and BSM particles can be described by a series of higher-dimensional effective operators with mass dimension $d>4[44,45]$. These operators are built out of SM fields and respect the symmetries of the SM. The SMEFT Lagrangian $\mathcal{L}_{\text {SMEFT }}$ is obtained by adding these $d$ dimensional operators $O_{i}^{(d)}$ together with corresponding Wilson coefficients $C_{i}^{(d)}$ to the SM Lagrangian $\mathcal{L}_{\mathrm{SM}}$. The $C_{i}^{(d)}$ encode the BSM couplings and, in order to be dimensionless, require a factor of $\Lambda^{4-d}$. The leading SMEFT contributions arise at dimension six:

$$
\mathcal{L}_{\mathrm{SMEFT}}=\mathcal{L}_{\mathrm{SM}}+\sum_{i} \frac{C_{i}^{(6)}}{\Lambda^{2}} O_{i}^{(6)}+\mathcal{O}\left(\Lambda^{-4}\right) .
$$

Contributions from odd-dimensional operators lead to lepton- and baryon-number violation $[46,47]$ and are neglected in this work. In the following, we employ the Warsaw basis [48] of dimension-six operators, and consider operators with gauge bosons

$$
\begin{aligned}
& O_{\varphi q}^{(1)}=\left(\varphi^{\dagger} i \overleftrightarrow{D}_{\mu} \varphi\right)\left(\bar{q}_{L} \gamma^{\mu} q_{L}\right), \quad O_{\varphi q}^{(3)}=\left(\varphi^{\dagger} i \overleftrightarrow{D}_{\mu}^{I} \varphi\right)\left(\bar{q}_{L} \tau^{I} \gamma^{\mu} q_{L}\right) \\
& O_{u B}=\left(\bar{q}_{L} \sigma^{\mu \nu} u_{R}\right) \tilde{\varphi} B_{\mu \nu}, \quad O_{u W}=\left(\bar{q}_{L} \sigma^{\mu \nu} \tau^{I} u_{R}\right) \tilde{\varphi} W_{\mu \nu}^{I}, \quad O_{u G}=\left(\bar{q}_{L} \sigma^{\mu \nu} T^{A} u_{R}\right) \tilde{\varphi} G_{\mu \nu}^{A}, \\
& O_{\varphi u}=\left(\varphi^{\dagger} i \overleftrightarrow{D}_{\mu} \varphi\right)\left(\bar{u}_{R} \gamma^{\mu} u_{R}\right)
\end{aligned}
$$

and semileptonic four-fermion operators

$$
\begin{aligned}
& O_{l q}^{(1)}=\left(\bar{l}_{L} \gamma_{\mu} l_{L}\right)\left(\bar{q}_{L} \gamma^{\mu} q_{L}\right), \quad O_{l q}^{(3)}=\left(\bar{l}_{L} \gamma_{\mu} \tau^{I} l_{L}\right)\left(\bar{q}_{L} \gamma^{\mu} \tau^{I} q_{L}\right), \quad O_{q e}=\left(\bar{q}_{L} \gamma_{\mu} q_{L}\right)\left(\bar{e}_{R} \gamma^{\mu} e_{R}\right), \\
& O_{e u}=\left(\bar{e}_{R} \gamma_{\mu} e_{R}\right)\left(\bar{u}_{R} \gamma^{\mu} u_{R}\right), \quad O_{l u}=\left(\bar{l}_{L} \gamma_{\mu} l_{L}\right)\left(\bar{u}_{R} \gamma^{\mu} u_{R}\right) .
\end{aligned}
$$

Here, $q_{L}, l_{L}$ are the quark and lepton $\mathrm{SU}(2)_{L}$ doublets, and $u_{R}, e_{R}$ the up-type quark and charged lepton $\mathrm{SU}(2)_{L}$ singlets, respectively. Flavor indices that exist for each $\mathrm{SM}$ 
fermion field are suppressed here for brevity but will be discussed in section 2.2. With $B_{\mu \nu}, W_{\mu \nu}^{I}$ and $G_{\mu \nu}^{A}$ we denote the gauge field strength tensors of $\mathrm{U}(1)_{Y}, \mathrm{SU}(2)_{L}$ and $\mathrm{SU}(3)_{C}$, respectively. $T^{A}=\lambda^{A} / 2$ and $\tau^{I} / 2$ are the generators of $\mathrm{SU}(3)_{C}$ and $\mathrm{SU}(2)_{L}$ in the fundamental representation with $A=1, \ldots, 8$ and $I=1,2,3$, and $\lambda^{A}$ and $\tau^{I}$ are the Gell-Mann and Pauli matrices, respectively. The SM Higgs doublet is denoted by $\varphi$ with its conjugate given as $\tilde{\varphi}=i \tau^{2} \varphi,\left(\varphi^{\dagger} i \overleftrightarrow{D}_{\mu} \varphi\right)=i \varphi^{\dagger}\left(D_{\mu} \varphi\right)-i\left(D_{\mu} \varphi^{\dagger}\right) \varphi$ and $\left(\varphi^{\dagger} i \overleftrightarrow{D}_{\mu}^{I} \varphi\right)=i \varphi^{\dagger} \tau^{I}\left(D_{\mu} \varphi\right)-i\left(D_{\mu} \varphi^{\dagger}\right) \tau^{I} \varphi$

Further dimension-six operators exist that contribute at subleading order to top-quark observables such as dipole operators $O_{d X}$ with $X=B, W, G$ and right-handed $b$ quarks, with contributions suppressed by $m_{b} / m_{t}$. We neglect those as well as all other SMEFT operators involving right-handed down-type quarks. Scalar and tensor operators $O_{\text {lequ }}^{(1 / 3)}$ are not included in our analysis since these operators do not give any relevant contributions at $\mathcal{O}\left(\Lambda^{-2}\right)$ for the interactions considered in this work [14, 40]. Contributions from fourquark operators to $t \bar{t} \gamma, t \bar{t} Z$ and $t \bar{t}$ production are neglected as $t \bar{t}$ production at the LHC is dominated by the $g g$ channel [8]. ${ }^{1}$ In addition we also neglect leptonic dipole operators, i.e., vertex corrections to lepton currents because they are severely constrained by $Z$-precision measurements [50].

Note that dipole operators are in general non-hermitian which allows for complexvalued Wilson coefficients. However, the dominant interference terms are proportional only to the real part of the coefficients. For the sake of simplicity, we thus assume all coefficients to be real-valued.

\section{$2.2 \quad$ Flavor and mass basis}

The dimension-six operators (2.2), (2.3) are given in the flavor basis. In general, quark mass and flavor bases are related by unitary transformations $S_{L / R}^{k}, k=u, d$,

$$
u_{L / R}^{i}=\left(S_{L / R}^{u}\right)_{i j} u_{L / R}^{\prime j}, \quad d_{L / R}^{i}=\left(S_{L / R}^{d}\right)_{i j} d_{L / R}^{\prime j},
$$

where $u^{\prime}$ and $d^{\prime}$ denote up- and down-type quarks in the mass basis, respectively, and $i, j=1,2,3$ are flavor indices. The CKM matrix $V$ is then given as

$$
V=\left(S_{L}^{u}\right)^{\dagger} S_{L}^{d}
$$

The rotation matrices of right handed quarks $S_{R}^{u / d}$ can simply be absorbed in the flavorbasis Wilson coefficient $C_{i}$, giving rise to coefficients in the mass basis, denoted by $\hat{C}_{i}$ [51]. In contrast, the flavor rotations $S_{L}^{u / d}$ of quark doublets relate different physical processes by $\mathrm{SU}(2)_{L}$-symmetry. Consider a contribution involving a doublet quark current with $\mathrm{SU}(2)_{L}$-singlet structure, i.e., the $C^{(1)} O^{(1)}$ terms with quark flavor indices restored. For

\footnotetext{
${ }^{1}$ Differential cross sections on the other hand are sensitive to four-fermion contributions [16]. Since bin-to-bin correlations are not available, yet important [49], we do not consider such observables in our fit.
} 
instance,

$$
\begin{aligned}
C_{\varphi q}^{(1) i j} O_{\varphi q}^{(1) i j} & =C_{\varphi q}^{(1) i j}\left(\varphi^{\dagger} i \overleftrightarrow{D}_{\mu} \varphi\right)\left(\bar{u}_{L}^{i} \gamma^{\mu} u_{L}^{j}+\bar{d}_{L}^{i} \gamma^{\mu} d_{L}^{j}\right) \\
& =C_{\varphi q}^{(1) i j}\left(\varphi^{\dagger} i \overleftrightarrow{D}_{\mu} \varphi\right)\left(\left(S_{L}^{u \dagger}\right)_{k i} \bar{u}_{L}^{\prime k} \gamma^{\mu}\left(S_{L}^{u}\right)_{j l} u_{L}^{\prime l}+\left(S_{L}^{d \dagger}\right)_{m i} \bar{d}_{L}^{\prime m} \gamma^{\mu}\left(S_{L}^{d}\right)_{j n} d_{L}^{\prime n}\right) \\
& =\hat{C}_{\varphi q}^{(1) k l}\left(\varphi^{\dagger} i \overleftrightarrow{D}_{\mu} \varphi\right)\left(\bar{u}_{L}^{\prime k} \gamma^{\mu} u_{L}^{\prime l}+V_{m k}^{\dagger} V_{l n} \bar{d}_{L}^{\prime m} \gamma^{\mu} d_{L}^{\prime n}\right) .
\end{aligned}
$$

Since we are interested in top-quark physics, in the last line we have chosen to work in the up-mass basis, the basis in which up-quark flavor and mass bases are identical and flavor mixing is entirely in the down-sector. Irrespective of this choice for the mass basis, $C_{\varphi q}^{(1) i j}$ induces in general contributions to both $u^{i}-u^{j}$ and $d^{i}-d^{j}$ transitions. In the up mass basis, $d^{i}-d^{j}$ transitions come with additional CKM-matrix elements. Contributions involving a doublet quark current with $\mathrm{SU}(2)_{L}$-triplet structure, i.e. the $C^{(3)} O^{(3)}$ terms have an additional minus sign between the up-sector and down-sector currents,

$$
C_{\varphi q}^{(3) i j} O_{\varphi q}^{(3) i j}=\hat{C}_{\varphi q}^{(3) k l}\left(\varphi^{\dagger} i \overleftrightarrow{D}_{\mu}^{3} \varphi\right)\left(\bar{u}_{L}^{\prime k} \gamma^{\mu} u_{L}^{\prime l}-V_{m k}^{\dagger} V_{l n} \bar{d}_{L}^{\prime m} \gamma^{\mu} d_{L}^{\prime n}\right)
$$

As a result, up-type and down-type quarks probe different combinations of $C^{(1)}$ and $C^{(3)}$, a feature recently also exploited in probing lepton flavor universality and conservation with processes involving neutrinos [52]. Further details on SMEFT coefficients and operators in the up-mass basis are given in appendix B and appendix $\mathrm{C}$, respectively.

In this analysis, we only consider contributions from (flavor basis) Wilson coefficients with third generation quarks, $\hat{C}_{i}^{33}$. Such hierarchies may arise in BSM scenarios with enhanced couplings to third-generation quarks, similar to the top-philic scenario discussed in ref. [11]. As can be seen in eqs. (2.6), (2.7), flavor mixing induces contributions to $d_{L}^{i} \rightarrow d_{L}^{j}$ transitions for $i \neq j$ with CKM suppressions $V_{t i} V_{t j}^{*}$, just like the SM. In this work, we include FCNC data from $b \rightarrow s$ transitions, while $s \rightarrow d$ transitions do presently not yield more significant constraints [19], and are not considered further. This leaves us with eleven real-valued SMEFT coefficients for the global fits

$$
\hat{C}_{u B}^{33}, \hat{C}_{u G}^{33}, \hat{C}_{u W}^{33}, \hat{C}_{\varphi q}^{(1) 33}, \hat{C}_{\varphi q}^{(3) 33}, \hat{C}_{\varphi u}^{33}, \hat{C}_{e u}^{33}, \hat{C}_{l u}^{33}, \hat{C}_{q e}^{33}, \hat{C}_{l q}^{(1) 33}, \hat{C}_{l q}^{(3) 33},
$$

defined in the up-mass basis.

Lepton universality does not have to be assumed for fits to present data since the bulk of the existing $b$-physics precision distributions is with muons. In the future, Belle II is expected to deliver both $b \rightarrow s e^{+} e^{-}$and $b \rightarrow s \mu^{+} \mu^{-}$distributions, and to shed light on the present hints that electrons and muons may be more different than thought [53]. In the far future, the $b \rightarrow s e^{+} e^{-}$results can be combined with $t \bar{t}$-production data from an $e^{+} e^{-}$collider; the muon ones could be combined with data from a muon collider, to improve the prospects for lepton flavor-specific fits. We also note that lepton flavor violating operators could also be included in the future. On the other hand, once data on dineutrino modes are included in the fit, assumptions on lepton flavor are in order, since the branching ratios are measured in a flavor-inclusive way:

$$
\mathcal{B}(b \rightarrow s \nu \bar{\nu})=\sum_{i, j} \mathcal{B}\left(b \rightarrow s \nu_{i} \bar{\nu}_{j}\right) .
$$




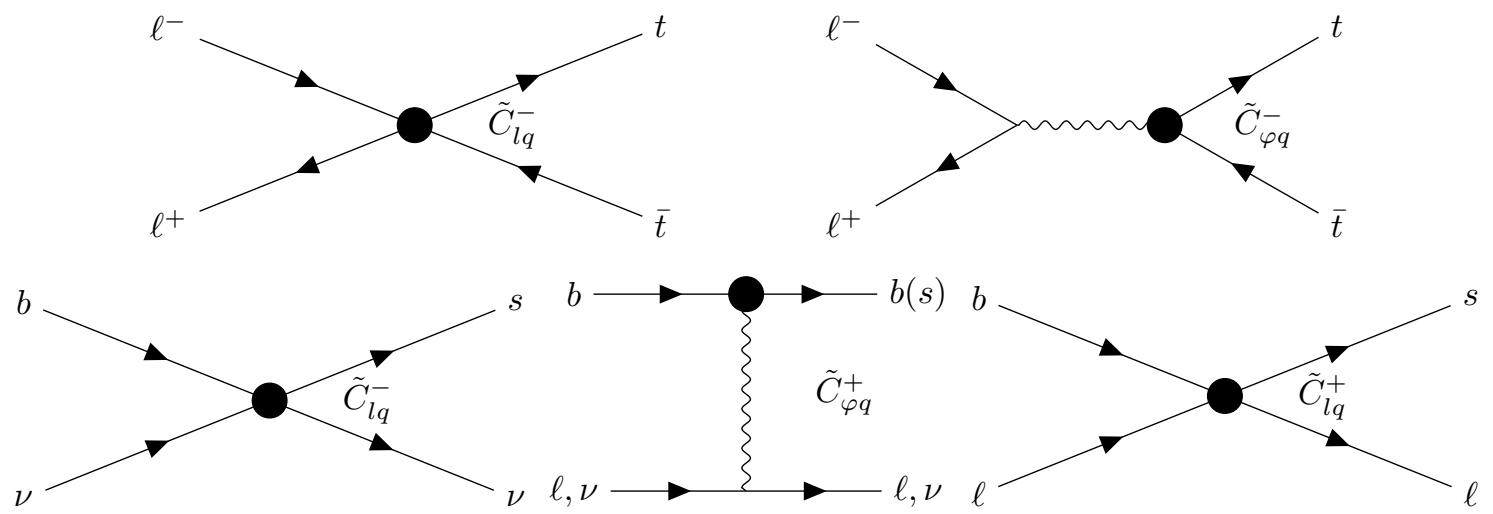

Figure 1. Sensitivities to $\tilde{C}_{l q}^{ \pm}$and $\tilde{C}_{\varphi q}^{ \pm}$, defined in (2.11), in top-quarks with charged leptons (upper row), and beauty with charged leptons and neutrinos (lower row). The black circles denote SMEFT operators, wavy lines are electroweak gauge bosons.

Universality dictates that the total dineutrino branching ratio is given by three times a flavor-specific one, $\mathcal{B}(b \rightarrow s \nu \bar{\nu})=3 \mathcal{B}\left(b \rightarrow s \nu_{i} \bar{\nu}_{i}\right)$. Here, $i$ is fixed, but could be any of the three flavors. We do assume universality when we include dineutrino modes in the fits to future data.

As is customary, in the following we use rescaled coefficients and drop the superscript for brevity

$$
\tilde{C}_{i}=\frac{v^{2}}{\Lambda^{2}} \hat{C}_{i}^{33}
$$

where $v=246 \mathrm{GeV}$ is the Higgs vacuum expectation value. To highlight $\mathrm{SU}(2)_{L}$ complementary between top and beauty, we introduce

$$
\tilde{C}_{l q}^{ \pm}=\tilde{C}_{l q}^{(1)} \pm \tilde{C}_{l q}^{(3)}, \quad \tilde{C}_{\varphi q}^{ \pm}=\tilde{C}_{\varphi q}^{(1)} \pm \tilde{C}_{\varphi q}^{(3)}
$$

The sensitivities are illustrated in figure 1.

\subsection{Matching and running: SMEFT and WET}

To constrain the Wilson coefficients of the SMEFT operators in eqs. (2.2) and (2.3) using $B$ physics measurements, the SMEFT Lagrangian has to be matched onto the WET Lagrangian, see appendix A for details. The procedure to compute BSM contributions at the scale $\mu_{b}$ in terms of coefficients given at a higher scale $\mu$ is described in detail in ref. [18] and adapted here. Throughout this work, we consider values for Wilson coefficients at the scale $\mu=1 \mathrm{TeV}$.

\subsubsection{SMEFT RGE}

The values of the Wilson coefficients depend on the energy scale $\mu$ of the process considered. The renormalization group equations (RGEs) allow to combine measurements at different scales in one analysis. The RGEs for eqs. (2.2) and (2.3) have been computed in refs. [5457]. We include these effects at one-loop level by applying the wilson [58] package. 


\subsubsection{Matching SMEFT onto WET}

Flavor rotations allow for contributions from $\hat{C}_{i}^{33}$ coefficients to $b \rightarrow s$ transitions whenever two $\mathrm{SU}(2)_{L}$ quark doublets are present in the operator. We obtain finite tree level contributions from $O_{\varphi q}^{(1)}, O_{\varphi q}^{(3)}, O_{l q}^{(1)}, O_{l q}^{(3)}$ and $O_{q e}$ to the WET coefficients of the semileptonic four-fermion operators $O_{9,10, L}$, defined in appendix A, as [51, 59]:

$$
\begin{aligned}
\Delta C_{9}^{\mathrm{tree}} & =\frac{\pi}{\alpha}\left[\tilde{C}_{l q}^{(1)}+\tilde{C}_{l q}^{(3)}+\tilde{C}_{q e}+\left(-1+4 \sin ^{2} \theta_{w}\right)\left(\tilde{C}_{\varphi q}^{(1)}+\tilde{C}_{\varphi q}^{(3)}\right)\right] \simeq \frac{\pi}{\alpha}\left[\tilde{C}_{l q}^{+}+\tilde{C}_{q e}\right], \\
\Delta C_{10}^{\mathrm{tree}} & =\frac{\pi}{\alpha}\left[-\tilde{C}_{l q}^{(1)}-\tilde{C}_{l q}^{(3)}+\tilde{C}_{q e}+\tilde{C}_{\varphi q}^{(1)}+\tilde{C}_{\varphi q}^{(3)}\right]=\frac{\pi}{\alpha}\left[-\tilde{C}_{l q}^{+}+\tilde{C}_{\varphi q}^{+}+\tilde{C}_{q e}\right], \\
\Delta C_{L}^{\mathrm{tree}} & =\frac{\pi}{\alpha}\left[\tilde{C}_{l q}^{(1)}-\tilde{C}_{l q}^{(3)}+\tilde{C}_{\varphi q}^{(1)}+\tilde{C}_{\varphi q}^{(3)}\right]=\frac{\pi}{\alpha}\left[\tilde{C}_{l q}^{-}+\tilde{C}_{\varphi q}^{+}\right],
\end{aligned}
$$

where $\sin ^{2} \theta_{w}=0.223$ denotes the weak mixing angle. We used for $\Delta C_{9}^{\text {tree }}$ in the second step the well-know suppression of $Z$-penguins due to the numerical smallness of the $Z$ 's vector coupling to charged leptons [60].

In addition to these dominant contributions, SMEFT operators induce contributions to WET dipole operators $O_{7,8}$, semileptonic operators $O_{9,10, L}$ and $|\Delta B|=2$ mixing at one-loop level [19, 51, 61-64]:

$$
\begin{aligned}
\Delta C_{7}^{\text {loop }}= & \frac{\sqrt{2} m_{t}}{m_{W}}\left[\tilde{C}_{u W} E_{7}^{u W}\left(x_{t}\right)+\tilde{C}_{u W}^{*} F_{7}^{u W}\left(x_{t}\right)+\frac{\cos \theta_{w}}{\sin \theta_{w}}\left(\tilde{C}_{u B} E_{7}^{u B}\left(x_{t}\right)+\tilde{C}_{u B}^{*} F_{7}^{u B}\left(x_{t}\right)\right)\right] \\
& +E_{7}^{\varphi q}\left(x_{t}\right) \tilde{C}_{\varphi q}^{(1)}+E_{7}^{\varphi q(3)}\left(x_{t}\right) \tilde{C}_{\varphi q}^{(3)}
\end{aligned}
$$

$$
\begin{aligned}
\Delta C_{8}^{\text {loop }}= & \frac{\sqrt{2} m_{t}}{m_{W}}\left[\tilde{C}_{u W} E_{8}^{u W}\left(x_{t}\right)+\tilde{C}_{u W}^{*} F_{8}^{u W}\left(x_{t}\right)-\frac{g}{g_{s}}\left(\tilde{C}_{u G} E_{8}^{u G}\left(x_{t}\right)+\tilde{C}_{u G}^{*} F_{8}^{u G}\left(x_{t}\right)\right)\right] \\
& +E_{8}^{\varphi q}\left(x_{t}\right) \tilde{C}_{\varphi q}^{(1)}+E_{8}^{\varphi q(3)}\left(x_{t}\right) \tilde{C}_{\varphi q}^{(3)}
\end{aligned}
$$

$$
\begin{aligned}
\Delta C_{9}^{\text {loop }}= & \sqrt{2} \frac{m_{t}}{m_{W}}\left[\left(\frac{Y_{u W}\left(x_{t}\right)}{\sin ^{2} \theta_{w}}-Z_{u W}\left(x_{t}\right)\right) \operatorname{Re}\left(\tilde{C}_{u W}\right)-\frac{\cos \theta_{w}}{\sin \theta_{w}} Z_{u B}\left(x_{t}\right) \operatorname{Re}\left(\tilde{C}_{u B}\right)\right] \\
& +\frac{1}{\sin ^{2} \theta_{w}}\left\{I_{1}\left(x_{t}\right)\left[\tilde{C}_{e u}+\tilde{C}_{l u}+\left(-1+4 \sin ^{2} \theta_{w}\right) \tilde{C}_{\varphi u}\right]+I_{2}\left(x_{t}\right)\left[\tilde{C}_{q e}+\tilde{C}_{l q}^{(1)}\right]\right. \\
& \left.+I^{l q}\left(x_{t}\right) \tilde{C}_{l q}^{(3)}+\left[\left(-1+4 \sin ^{2} \theta_{w}\right) I_{2}\left(x_{t}\right) \tilde{C}_{\varphi q}^{(1)}+I_{1}^{\varphi q}\left(x_{t}\right) \tilde{C}_{\varphi q}^{(3)}\right]\right\}
\end{aligned}
$$

$$
\begin{aligned}
\Delta C_{10}^{\text {loop }}= & -\frac{\sqrt{2}}{\sin ^{2} \theta_{w}} \frac{m_{t}}{m_{W}} Y_{u W}\left(x_{t}\right) \operatorname{Re}\left(\tilde{C}_{u W}\right) \\
& +\frac{1}{\sin ^{2} \theta_{w}}\left\{I_{1}\left(x_{t}\right)\left[\tilde{C}_{e u}-\tilde{C}_{l u}+\tilde{C}_{\varphi u}\right]+I_{2}\left(x_{t}\right)\left[\tilde{C}_{q e}-\tilde{C}_{l q}^{(1)}\right]\right. \\
& \left.-I^{l q}\left(x_{t}\right) \tilde{C}_{l q}^{(3)}+\left[I_{2}\left(x_{t}\right)\left(x_{t}\right) \tilde{C}_{\varphi q}^{(1)}+I_{2}^{\varphi q}\left(x_{t}\right) \tilde{C}_{\varphi q}^{(3)}\right]\right\}
\end{aligned}
$$




$$
\begin{aligned}
\Delta C_{L}^{\mathrm{loop}}= & I_{u W}^{\nu} \operatorname{Re}\left(\tilde{C}_{u W}\right)+I_{\varphi q}^{\nu(1)} \tilde{C}_{\varphi q}^{(1)}+I_{\varphi q}^{\nu(3)} \tilde{C}_{\varphi q}^{(3)}+I_{l u}^{\nu}\left(\tilde{C}_{\varphi u}+\tilde{C}_{l u}\right)+I_{l q}^{\nu(1)} \tilde{C}_{l q}^{(1)}+I_{l q}^{\nu(3)} \tilde{C}_{l q}^{(3)} \\
\Delta C_{1, t t}^{\text {mix, loop }}= & +\sqrt{2} \frac{m_{t}}{m_{W}} \operatorname{Re}\left(\tilde{C}_{u W}\right) \frac{9 x_{t}}{4}\left(\frac{x_{t}+1}{\left(x_{t}-1\right)^{2}}-\frac{2 x_{t}}{\left(x_{t}-1\right)^{3}} \log x_{t}\right)+4 S_{0}\left(x_{t}\right) \tilde{C}_{\varphi q}^{(3)} \\
& +H_{1}\left(x_{t}\right) \tilde{C}_{\varphi q}^{(1)}+H_{2}\left(x_{t}\right) \tilde{C}_{\varphi q}^{(3)}
\end{aligned}
$$

which are present also in absence of CKM-mixing, and with $x_{t}=m_{t}^{2} / m_{W}^{2}$. Explicit expressions for the $x_{t}$-dependent functions can be found in refs. [19, 51, 61-64]. For completeness, we also give these functions in appendix D.

Note that there is sensitivity, although only at the one-loop level, to the semileptonic operators with up-type singlet quarks, $O_{e u}$ and $O_{l u}$. The numerical values of the matching conditions at $\mu_{W}=m_{W}$ are computed with wilson following ref. [62] and are provided in appendix E. In the actual analysis, RGE effects in SMEFT and WET are taken into account as well.

\subsubsection{WET RGE}

We employ flavio [65] and wilson to compute the values of the SM and BSM contributions at the scale $\mu_{b}$.

\section{Observables}

In this section we give details on how theory predictions and distributions for topobservables (section 3.1), $Z \rightarrow b \bar{b}$ transitions (section 3.2), and $B$-physics (section 3.3) are obtained, and discuss the sensitivities of the observables to SMEFT coefficients (section 3.4).

\subsection{Top-quark observables}

We employ the Monte Carlo (MC) generator MADGraph5_aMC@NLO [66] to compute the $t \bar{t}, t \bar{t} \gamma$ and $t \bar{t} Z$ production cross sections at the LHC and the $t \bar{t}$ production cross section and the forward-backward symmetry at CLIC in LO QCD. The cross sections can be parametrized in terms of the Wilson coefficients as

$$
\sigma=\sigma^{\mathrm{SM}}+\sum_{i} \tilde{C}_{i} \sigma_{i}^{\text {int. }}+\sum_{i \leq j} \tilde{C}_{i} \tilde{C}_{j} \sigma_{i j}^{\mathrm{BSM}},
$$

where $\sigma_{i}^{\text {int. }}$ and $\sigma_{i j}^{\mathrm{BSM}}$ denote interference terms between SM and dimension-six operators and purely BSM terms, respectively. The forward-backward asymmetry is defined as

$$
A_{\mathrm{FB}}=\frac{\sigma_{\mathrm{FB}}}{\sigma}, \quad \sigma_{\mathrm{FB}}=\int_{-1}^{1} \mathrm{~d} \cos \theta \operatorname{sign}(\cos \theta) \frac{d \sigma}{d \cos \theta},
$$

where $\theta$ denotes the angle between the three-momenta of the top quark and the positron in the center-of-mass frame. BSM contributions in both numerator and denominator are parametrized according to eq. (3.1). 
To obtain $\sigma_{i}^{\text {int. }}$ and $\sigma_{i j}^{\text {BSM }}$ we utilize the dim6top_Lo UFO model [11]. For the computation of the fiducial cross sections of $t \bar{t} \gamma$ production we generate samples as a $2 \rightarrow 7$ process including BSM contributions in the top-quark decay. The fiducal acceptances are obtained by showering the events with PYTHIA8 [67] and performing an event selection at particle level with MadAnalysis [68-70]. For the jet clustering we apply the anti- $k_{t}$ algorithm [71] with radius parameter $R=0.4$ using Fast Jet [72]. The computation is discussed in detail in ref. [18].

We compute the helicity fractions according to ref. [73] with the difference that we also include quadratic contributions. In our analysis, we consider only $O_{u W}$ as only this operator gives contributions $\mathcal{O}\left(\Lambda^{-2}\right)$ that are not suppressed by a factor $m_{b} / m_{t}$. The top-quark decay width is computed following ref. [74] including quadratic contributions.

\section{$3.2 \quad Z b \bar{b}$ observables}

To compute $Z \rightarrow b \bar{b}$ observables we employ MADGraPh5_aMC@NLO together with the dim6top_LO UFO model for both the forward-backward asymmetry $A_{\mathrm{FB}}^{0, b}$ and the ratio of partial widths for $Z \rightarrow f \bar{f}$

$$
R_{b}=\frac{\Gamma_{b \bar{b}}}{\Gamma_{\text {had }}}, \quad \Gamma=\Gamma^{\mathrm{SM}}+\sum_{i} \tilde{C}_{i} \Gamma_{i}^{\mathrm{int}}+\sum_{i \leq j} \tilde{C}_{i} \tilde{C}_{j} \Gamma_{i j}^{\mathrm{BSM}} .
$$

BSM contributions to $A_{\mathrm{FB}}^{0, b}$ are computed using eq. (3.2), and for $R_{b}$ we include BSM contributions in both numerator and denominator.

\section{3 $\quad B$-physics observables}

For observables in $b \rightarrow s \gamma$ and $b \rightarrow s \ell^{+} \ell^{-}$transitions we employ flavio together with the wilson package to compute the BSM contributions in terms of $\Delta \bar{C}_{i}^{(0)}\left(\mu_{W}\right)$ at the scale $\mu_{W}=m_{W}$. For $b \rightarrow s \nu \bar{\nu}$ the Wilson coefficient $C_{L}$ does not run. BSM contributions are considered at LO in $\alpha_{s}$ and run with wilson in the WET basis from the scale $\mu_{W}$ to $\mu_{b}$, at which the observables are computed. To compute the observables for different values of the SMEFT Wilson coefficients $\tilde{C}_{i}$, they are run from the scale $\mu$ to $\mu_{W}$ and matched onto the WET basis according to eqs. (2.12)-(2.16).

Branching ratios of $b \rightarrow s \nu \bar{\nu}$ transitions are computed via [59]

$$
\mathrm{BR}\left(B \rightarrow K^{(*)} \nu \bar{\nu}\right)=\mathrm{BR}\left(B \rightarrow K^{(*)} \nu \bar{\nu}\right)_{\mathrm{SM}} \frac{\left|\Delta C_{L}\left(\mu_{b}\right)-C_{L}\left(\mu_{b}\right)_{\mathrm{SM}}\right|}{C_{L}\left(\mu_{b}\right)_{\mathrm{SM}}},
$$

where

$$
\begin{aligned}
& \mathrm{BR}\left(B^{+} \rightarrow K^{+} \nu \bar{\nu}\right)_{\mathrm{SM}}=(4.0 \pm 0.5) \times 10^{-6}, \\
& \mathrm{BR}\left(B^{0} \rightarrow K^{0 *} \nu \bar{\nu}\right)_{\mathrm{SM}}=(9.2 \pm 1.0) \times 10^{-6},
\end{aligned}
$$

and $C_{L}\left(\mu_{b}\right)_{\mathrm{SM}}=\frac{X_{s}}{\sin ^{2} \theta_{w}}$ with $X_{s}=1.469 \pm 0.017$, and lepton flavor universality is assumed.

We also consider the $B_{s}-\bar{B}_{s}$ mass difference $\Delta M_{s}$, which can be computed as [75]

$$
\Delta M_{s}=\Delta M_{s}^{\mathrm{SM}}\left|1+\frac{\Delta C_{1, t t}^{\operatorname{mix}}\left(\mu_{W}\right)}{S_{0}\left(x_{t}\right)}\right|,
$$

where $S_{0}$ denotes the Inami-Lim function. We employ the SM value $\Delta M_{s}^{\text {SM }}=$ $\left(18.4_{-1.2}^{+0.7}\right) \mathrm{ps}^{-1}[75]$. 


\begin{tabular}{|cc|c|c|}
\hline Process & Observable & Two-fermion operators & Four-fermion operators \\
\hline$p p \rightarrow t \bar{t}$ & $\sigma^{\text {inc }}$ & $\tilde{C}_{u G}$ & - \\
$p p \rightarrow t \bar{t} \gamma$ & $\sigma^{\text {fid }}$ & $\tilde{C}_{u B}, \tilde{C}_{u W}, \tilde{C}_{u G}$ & - \\
$p p \rightarrow t \bar{t} Z$ & $\sigma^{\text {inc }}$ & $\tilde{C}_{u B}, \tilde{C}_{u W}, \tilde{C}_{u G}, \tilde{C}_{\varphi q}^{-}, \tilde{C}_{\varphi u}$ & - \\
$t \rightarrow b W$ & $F_{0, L}$ & $\tilde{C}_{u W}$ & - \\
Top decay & $\Gamma_{t}$ & $\tilde{C}_{\varphi q}^{(3)}, \tilde{C}_{u W}$ & - \\
\hline$Z \rightarrow b \bar{b}$ & $A_{F B}^{b}, R_{b}, \sigma_{\mathrm{had}}$ & $\tilde{C}_{\varphi q}^{+}$ & - \\
\hline$b \rightarrow s \gamma$ & $\mathrm{BR}$ & {$\left[\tilde{C}_{u B}\right],\left[\tilde{C}_{u W}\right],\left\{\tilde{C}_{u G}\right\},\left[\tilde{C}_{\varphi q}^{(3)}\right]$} & $\tilde{C}_{l q}^{+(*)}, \tilde{C}_{q e}^{(*)}$ \\
$b \rightarrow s \ell^{+} \ell^{-}$ & $\mathrm{BR}, A_{\mathrm{FB}}, P_{i}^{(\prime)}$, & {$\left[\tilde{C}_{u B}\right],\left[\tilde{C}_{u W}\right],\left\{\tilde{C}_{u G}\right\}, \tilde{C}_{\varphi q}^{+(*)},\left[\tilde{C}_{\varphi q}^{(3)}\right]$} & $\tilde{C}_{l q}^{-(*)}$ \\
$b \rightarrow s \nu \bar{\nu}$ & $\mathrm{BR}$ & $\tilde{C}_{\varphi q}^{+(* *)}$ & - \\
Mixing & $\Delta M_{s}$ & {$\left[\tilde{C}_{u W}\right],\left\{\tilde{C}_{u G}\right\},\left[\tilde{C}_{\varphi q}^{(1)}\right],\left[\tilde{C}_{\varphi q}^{(3)}\right]$} & - \\
\hline$e^{+} e^{-} \rightarrow t \overline{\mathrm{BR}} / d q^{2}$ & $\sigma, A_{\mathrm{FB}}$ & $\tilde{C}_{u B}, \tilde{C}_{u W},\left\{\tilde{C}_{u G}\right\}, \tilde{C}_{\varphi q}^{-}, \tilde{C}_{\varphi u}$ & $\tilde{C}_{e u}, \tilde{C}_{q e}, \tilde{C}_{l u}, \tilde{C}_{l q}^{-}$ \\
\hline
\end{tabular}

Table 1. SMEFT contributions to the observables included in the fit. Coefficients without parentheses arise at tree level. Coefficients marked as $\left[\tilde{C}_{i}\right]$ contribute only at one-loop level to $B$ physics observables while contributions marked as $\left\{\tilde{C}_{i}\right\}$ are induced by SMEFT and WET running at $\mathcal{O}\left(\alpha_{s}\right)$ only. Coefficients $\tilde{C}_{i}^{(*)}$ and $\tilde{C}_{i}^{(* *)}$ receive contributions at one-loop level that change their tree-level definitions, see eqs. (3.7), (3.8).

\subsection{Sensitivity to BSM contributions}

In table 1 we summarize which linear combinations of SMEFT Wilson coefficients contribute to each observable. Contributions denoted in square brackets $\left[\tilde{C}_{i}\right]$ are induced at one-loop level only, while those written as $\left\{\tilde{C}_{i}\right\}$ contribute only via RGE evolution. Tree-level coefficients marked with an asterisk receive additional contribution at one-loop level, which are suppressed by at least one order of magnitude, see eqs. (3.7), (3.8) and appendix E for details.

Total cross sections of the top-quark production channels, the top-quark decay width, and the helicity fractions measured at the LHC allow to test six coefficients of the operators in eq. (2.2), namely, $\tilde{C}_{u B}, \tilde{C}_{u W}, \tilde{C}_{u G}, \tilde{C}_{\varphi u}, \tilde{C}_{\varphi q}^{(1)}$, and $\tilde{C}_{\varphi q}^{(3)} \cdot{ }^{2}$ While $t \bar{t} Z$ production is only sensitive to the linear combination $\tilde{C}_{\varphi q}^{-}$(see eq. (2.11)), the total decay width is sensitive to $\tilde{C}_{\varphi q}^{(3)}$. Thus, including data from top-quark decay allows to test $\tilde{C}_{\varphi q}^{(1)}$ and $\tilde{C}_{\varphi q}^{(3)}$ individually. Note that contributions from $\tilde{C}_{u G}$ to any of the $B$-physics and lepton collider observables we consider arise only from RGE evolution at $\mathcal{O}\left(\alpha_{s}\right)$ and mixing.

Observables of $Z \rightarrow b \bar{b}$ decay are sensitive to $\tilde{C}_{\varphi q}^{+}$, and the other operators considered here do not contribute to this process.

\footnotetext{
${ }^{2}$ At the LHC, single top production is sensitive to these coefficients as well. However, bin-to-bin correlations are not publicly available and we therefore do not consider these observables, see also footnote 1.
} 
Including $b \rightarrow s$ observables allows to put new and stronger constraints on SMEFT coefficients. The interplay of $b \rightarrow s \gamma$ transitions with $t \bar{t} \gamma$ has been worked out in [18]. BSM contributions to the former are induced at one-loop level by $\tilde{C}_{u B}, \tilde{C}_{u W}, \tilde{C}_{u G}$, and $\tilde{C}_{\varphi q}^{(3)}$.

For $b \rightarrow s \ell^{+} \ell^{-}$transitions, tree level contributions to $\Delta C_{9,10}$ arise from $\tilde{C}_{\varphi q}^{+}, \tilde{C}_{l q}^{+}$, defined in eq. (2.11), and $\tilde{C}_{q e}$. The latter cancels, however, in the left-chiral combination $\Delta C_{9}-\Delta C_{10}$, which is the one that gives the dominant interference term in semileptonic $B$ decays with the SM. We therefore expect only little sensitivity to $\tilde{C}_{q e}$ from these modes. On the other hand, this highlights the importance of $B_{s} \rightarrow \mu \mu$, which is sensitive to $C_{10}$ only. At one-loop level, all eleven SMEFT operators considered here contribute to $\Delta C_{9,10}$ $\left(\tilde{C}_{u G}\right.$ only via mixing). In the case of $\tilde{C}_{\varphi q}^{(1)}, \tilde{C}_{\varphi u}, \tilde{C}_{l q}^{(1)}, \tilde{C}_{l q}^{(3)}, \tilde{C}_{l u}, \tilde{C}_{q e}, \tilde{C}_{e u}$, and partially $\tilde{C}_{\varphi q}^{(3)}$, these contributions can simply be absorbed by redefining the fit degrees of freedom

$$
\begin{aligned}
\tilde{C}_{l q}^{+(*)} & =\tilde{C}_{l q}^{+}+\frac{\alpha}{\pi \sin ^{2} \theta_{w}}\left(I_{1}\left(x_{t}\right) \tilde{C}_{l u}+I_{2}\left(x_{t}\right) \tilde{C}_{l q}^{+}\right), \\
\tilde{C}_{q e}^{(*)} & =\tilde{C}_{q e}+\frac{\alpha}{\pi \sin ^{2} \theta_{w}}\left(I_{1}\left(x_{t}\right) \tilde{C}_{e u}+I_{2}\left(x_{t}\right) \tilde{C}_{q e}\right), \\
\tilde{C}_{\varphi q}^{+(*)} & =\tilde{C}_{\varphi q}^{+}+\frac{\alpha}{\pi \sin ^{2} \theta_{w}}\left(I_{1}\left(x_{t}\right) \tilde{C}_{\varphi u}+I_{2}\left(x_{t}\right) \tilde{C}_{\varphi q}^{+}\right) .
\end{aligned}
$$

Numerically, these loop-level corrections are typically below percent-level compared to treelevel contributions. For the remaining contributions from $\tilde{C}_{\varphi q}^{(3)}, \tilde{C}_{u B}, \tilde{C}_{u W}$ (and $\tilde{C}_{u G}$ ) to $\Delta C_{9,10}$ such a redefinition is not possible and additional degrees of freedom arise. However, these remaining contributions to $\Delta C_{9,10}$ are at least one order of magnitude smaller than the tree-level ones.

At tree level, $b \rightarrow s \nu \bar{\nu}$ transitions are sensitive to $\tilde{C}_{\varphi q}^{+}+\tilde{C}_{l q}^{-}$. Additional loop-level contributions by $\tilde{C}_{u W}, \tilde{C}_{\varphi q}^{(1)}, \tilde{C}_{\varphi q}^{(3)}, \tilde{C}_{\varphi u}, \tilde{C}_{l q}^{(1)}, \tilde{C}_{l q}^{(3)}$ and $\tilde{C}_{l u}$ can be absorbed into $\tilde{C}_{\varphi q}^{+(* *)}$ and $\tilde{C}_{l q}^{-(*)}$ :

$$
\begin{aligned}
\tilde{C}_{\varphi q}^{+(* *)} & =\tilde{C}_{\varphi q}^{+}-\sqrt{2} \frac{\alpha m_{t}}{\pi m_{W}} I_{u W}^{\nu} \tilde{C}_{u W}-\frac{\alpha}{\pi} I_{\varphi q}^{\nu(3)}\left(x_{t}\right) \tilde{C}_{\varphi q}^{(3)}+\frac{\alpha}{\pi}\left(I_{l u}^{\nu}\left(x_{t}\right) \tilde{C}_{\varphi u}+I_{\varphi q}^{\nu(1)} \tilde{C}_{\varphi q}^{(1)}\right), \\
\tilde{C}_{l q}^{-(*)} & =\tilde{C}_{l q}^{-}+\frac{\alpha}{\pi}\left(I_{l u}^{\nu}\left(x_{t}\right) \tilde{C}_{l u}+I_{l q}^{\nu(1)}\left(x_{t}\right) \tilde{C}_{l q}^{(1)}\right)+\frac{\alpha}{\pi} I_{l q}^{\nu(3)}\left(x_{t}\right) \tilde{C}_{l q}^{(3)} .
\end{aligned}
$$

Dineutrino observables depend only on the sum of these coefficients. Meson mixing is sensitive at one-loop level to $\tilde{C}_{u W}, \tilde{C}_{\varphi q}^{(1)}$, and $\tilde{C}_{\varphi q}^{(3)}$ while contributions from $\tilde{C}_{u G}$ arise only through SMEFT $\mathcal{O}\left(\alpha_{s}\right)$ RGE evolution. Electroweak RGE effects in $B$ physics [76] as well as in top-quark physics are included in the numeric fits but are not shown here for clarity.

In summary, while all SMEFT coefficients contribute to the $B$ physics observables considered, these effects are mostly induced at one-loop level and thus naturally suppressed. Notable exceptions are tree-level contributions from $\tilde{C}_{\varphi q}^{+}, \tilde{C}_{l q}^{+}, \tilde{C}_{q e}$, and $\tilde{C}_{l q}^{-}+\tilde{C}_{\varphi q}^{+}$. In addition, $\tilde{C}_{u B}$ is important as it contributes with sizable coefficient to $\Delta C_{7}$ [18]. Thus, we expect that $B$ physics data constrains these SMEFT-coefficients rather strongly, and the others much less.

Measurements of top-quark pair production cross sections and the forward-backward asymmetry at a lepton collider are sensitive to four linear combinations of two-fermion 


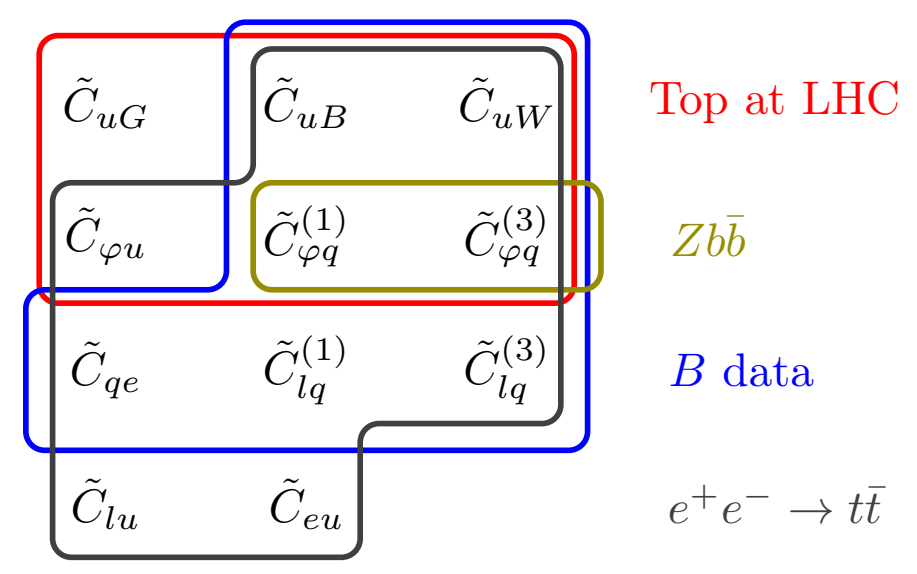

Figure 2. Schematic overview of dominant contributions from SMEFT coefficients to the different sets of observables considered in this work. Subleading contributions at one-loop level as well as mixing-induced ones from $\tilde{C}_{u G}$ are neglected.

operators $\tilde{C}_{u B}, \tilde{C}_{u W}, \tilde{C}_{\varphi q}^{-}$, and $\tilde{C}_{\varphi u}$. The sensitivity to $\tilde{C}_{u G}$ is smaller because contributions arise only through RGE evolution. While these coefficients affect the $t t Z$ and $t t \gamma$ vertex, four-fermion operators can also contribute in following linear combinations: $\tilde{C}_{l q}^{-}$, $\tilde{C}_{q e}, \tilde{C}_{e u}$, and $\tilde{C}_{l u}$. Thus, combining $\ell^{+} \ell^{-} \rightarrow t \bar{t}$ observables with top-quark ones at LHC and $B$ physics observables allows to test the complete 11-dimensional parameter space. In particular, coefficients $\tilde{C}_{e u}$ and $\tilde{C}_{l u}$ remain only poorly constrained by Belle II and the HLLHC. A summary of the dominant contributions to the different observables is illustrated in figure 2.

\section{Fits to present data}

We employ EFTfitter [77], which is based on the Bayesian Analysis Toolkit - BAT.jl [78], to constrain the Wilson coefficients in a Bayesian interpretation. We include systematic and statistical experimental and SM theory uncertainties. All uncertainties on the measured observables are assumed to be Gaussian distributed. The procedure of our fit is detailed in our previous analyses in refs. [18, 49], and is based on ref. [77].

BSM contributions are parametrized as in (3.1), which includes quadratic dimensionsix terms. While these purely BSM contributions are formally of higher order in the EFT expansion, $\mathcal{O}\left(\Lambda^{-4}\right)$, it has been shown $[12,49]$ that the inclusion of such quadratic terms has only a negligible effect on constraints of coefficients for which the linear term in the EFT expansion gives the dominant contribution, as expected in regions where the EFT is valid.

We include measurements of observables from both top-quark pair production processes and top-quark decay at the LHC, $Z \rightarrow b \bar{b}$ transitions, and $b \rightarrow s$ transitions from different experiments. Measurements of the same observable from different experiments can in principle be correlated [79]. Correlations are included as long as they are provided, comprising mainly bin-to-bin correlations and correlations between the $W$ boson helicity fractions. Unknown correlations can affect the result of the fit significantly [49]. Therefore, 

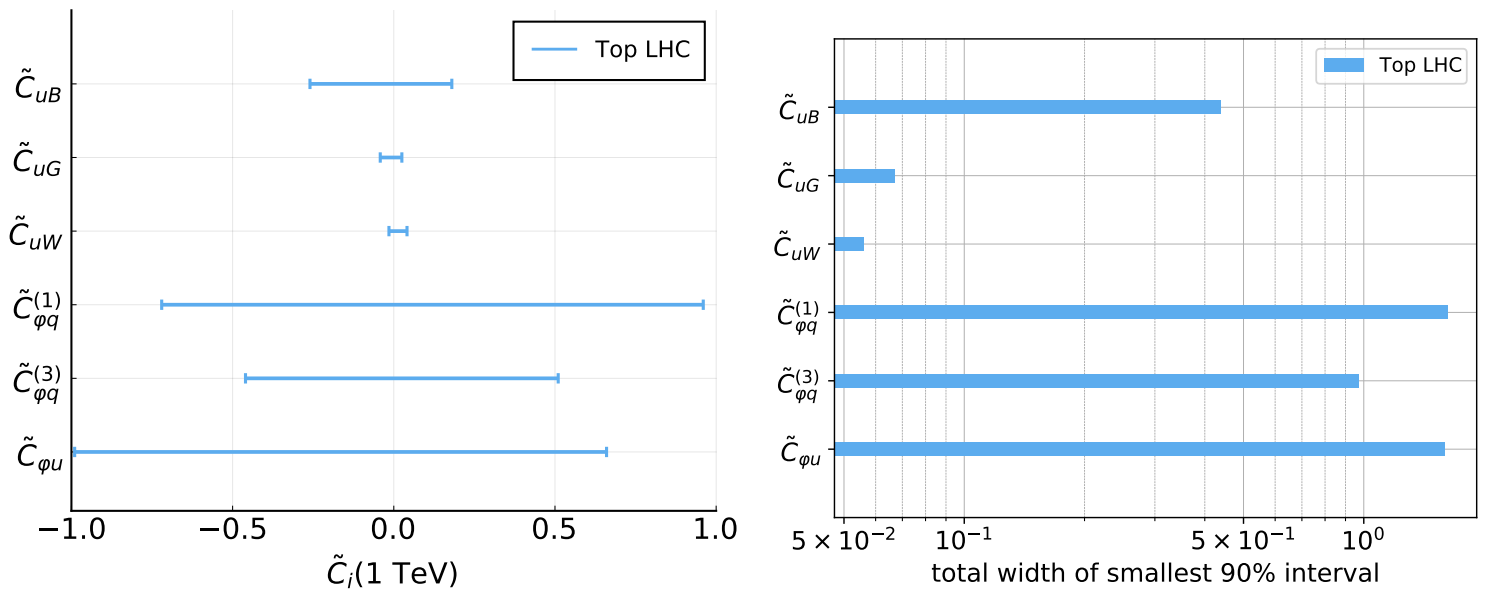

Figure 3. Constraints on SMEFT Wilson coefficients $\tilde{C}_{i}$ from the measurements of top-quark observables in table 2. Shown are the marginalized smallest intervals containing $90 \%$ posterior probability (left) and the total width of these intervals (right) obtained in a fit of six coefficients to top-quark data. For all coefficients we choose a uniform distribution in the interval $-1 \leq \tilde{C}_{i} \leq 1$ as the prior probability.

we follow a strategy similar to the ones of refs. $[14,16]$ and include only the most precise measurement of an observable in the fit. Especially, if no complete correlation matrices for differential distributions are given by the experiments, we do not include these measurements in the analysis. For $B$ physics observables, a variety of measurements have been combined by the Heavy Flavour Averaging Group (HFLAV) [80]. Wherever possible, we include their averaged experimental values in our analysis. For all remaining unknown correlations between different observables, we make the simplifying assumptions that the measurements included in the fit are uncorrelated.

We work out current constraints from top-quark measurements in section 4.1, from $Z \rightarrow b \bar{b}$ data in section 4.2 , from $B$-physics in section 4.3 , and perform a global analysis in section 4.4.

\subsection{Current constraints from top-quark measurements at the LHC}

In table 2 we summarize the measurements and the corresponding SM predictions of the top-quark observables at the LHC included in the fit. This dataset comprises measurements of fiducal cross sections $\sigma^{\text {fid }}(t \bar{t} \gamma, 1 \ell)\left(\sigma^{\text {fid }}(t \bar{t} \gamma, 2 \ell)\right)$ of $t \bar{t} \gamma$ production in the single lepton (dilepton) channel, inclusive cross sections $\sigma^{\text {inc }}(t \bar{t})$ and $\sigma^{\text {inc }}(t \bar{t} Z)$ of $t \bar{t}$ and $t \bar{t} Z$ production, respectively, measurements of the $W$ boson helicity fractions $F_{0, L}$, and a measurement of the total top-quark decay width $\Gamma_{t}$. The SM predictions for $t \bar{t} \gamma$ cross sections include NLO QCD corrections refs. [81, 82], while predictions for $t \bar{t} Z$ cross sections are computed at NLO QCD including electroweak corrections [84-86]. For $t \bar{t}$ production, the SM prediction at NNLO QCD is taken from ref. [87], and has been computed following ref. [88]. Predictions for helicity fractions and the total decay width include NNLO QCD corrections [90, 92].

In figure 3 we give constraints on SMEFT Wilson coefficients detailed in table 1 obtained in a fit of six coefficients to the data in table 2 . The strongest constraints are found 


\begin{tabular}{|ccccccc|}
\hline Process & Observable & $\sqrt{s}$ & Int. luminosity & Experiment & Ref. & SM ref. \\
\hline$t \bar{t} \gamma$ & $\sigma^{\text {fid }}(t \bar{t} \gamma, 1 \ell), \sigma^{\text {fid }}(t \bar{t} \gamma, 2 \ell)$ & $13 \mathrm{TeV}$ & $36.1 \mathrm{fb}^{-1}$ & ATLAS & {$[81]$} & {$[81,82]$} \\
\hline$t \bar{t} Z$ & $\sigma^{\text {inc }}(t \bar{t} Z)$ & $13 \mathrm{TeV}$ & $77.5 \mathrm{fb}^{-1}$ & CMS & {$[83]$} & {$[84-86]$} \\
\hline$t \bar{t}$ & $\sigma^{\text {inc }}(t \bar{t})$ & $13 \mathrm{TeV}$ & $36.1 \mathrm{fb}^{-1}$ & ATLAS & {$[87]$} & {$[88]$} \\
& $F_{0}, F_{L}$ & $8 \mathrm{TeV}$ & $20.2 \mathrm{fb}^{-1}$ & ATLAS & {$[89]$} & {$[90]$} \\
& $\Gamma_{t}$ & $8 \mathrm{TeV}$ & $20.2 \mathrm{fb}^{-1}$ & ATLAS & {$[91]$} & {$[92]$} \\
\hline
\end{tabular}

Table 2. Considered observables for top-quark processes at the LHC and references for the corresponding measurements and SM calculations.

for $\tilde{C}_{u G}$ and $\tilde{C}_{u W}$, which are at the level of $\mathcal{O}\left(10^{-2}\right)$ and stem from measurements of $t \bar{t}$ production cross sections and the $W$ boson helicity fractions, respectively. Constraints on $\tilde{C}_{u B}$, which are dominated by $t \bar{t} \gamma$ measurements, are at the level of $\mathcal{O}\left(10^{-1}\right)$. Including measurements of the top-quark decay width allows constraining $\tilde{C}_{\varphi q}^{(3)}$ to a level of $\mathcal{O}(1)$. Given present data, results are limited by large experimental uncertainties. Both $\tilde{C}_{\varphi q}^{(1)}$ and $\tilde{C}_{\varphi u}$ remain almost unconstrained by the measurements of $t \bar{t} Z$ production due to a strong correlation between their contributions and larger uncertainties of measurements and theory predictions.

\subsection{Constraints from $Z b b$ measurements}

Precision measurements of $Z$ pole observables have been performed at LEP 1 and SLC, and the results are collected in ref. [50]. In our analysis, we focus on those that are sensitive to BSM contributions which affect the $Z b \bar{b}$ vertex. The measurements included are those of the forward-backward asymmetry and the ratio of partial widths for $Z \rightarrow f \bar{f}$ [93]

$$
A_{F B}^{0, b \operatorname{Exp}}=0.0996 \pm 0.0016, \quad R_{b}^{\operatorname{Exp}}=0.21629 \pm 0.00066 .
$$

The corresponding SM values are given as [50, 93]

$$
A_{F B}^{0, b \mathrm{SM}}=0.1030 \pm 0.0002, \quad R_{b}^{\mathrm{SM}}=0.21581 \pm 0.00002 .
$$

These observables are sensitive to BSM contributions from $\tilde{C}_{\varphi q}^{+}$, which alter the $Z b \bar{b}$ vertex, and allow to derive strong constraints on this coefficient. The results of a fit of one $\left(\tilde{C}_{\varphi q}^{+}\right)$ and two $\left(\tilde{C}_{\varphi q}^{(1)}, \tilde{C}_{\varphi q}^{(3)}\right)$ coefficients to $Z b \bar{b}$ data are shown in figure 4 . As can be seen, this dataset strongly constrains $\tilde{C}_{\varphi q}^{+}$to a level of $\mathcal{O}\left(10^{-3}\right)$. Due to the deviations from the SM present in $A_{F B}^{0, b}$ we observe deviations of about $2 \sigma$ in $\tilde{C}_{\varphi q}^{+}$. Considering results in the $\tilde{C}_{\varphi q}^{(1)}-\tilde{C}_{\varphi q}^{(3)}$ plane we find, as expected, strong correlations, and only a very small slice of the two-dimensional parameter space is allowed by present data.

\subsection{Current constraints from $B$ physics measurements}

In table 3 we give the $B$ physics observables and the corresponding references of the measurements and SM predictions considered in our fit. This dataset includes both inclusive 

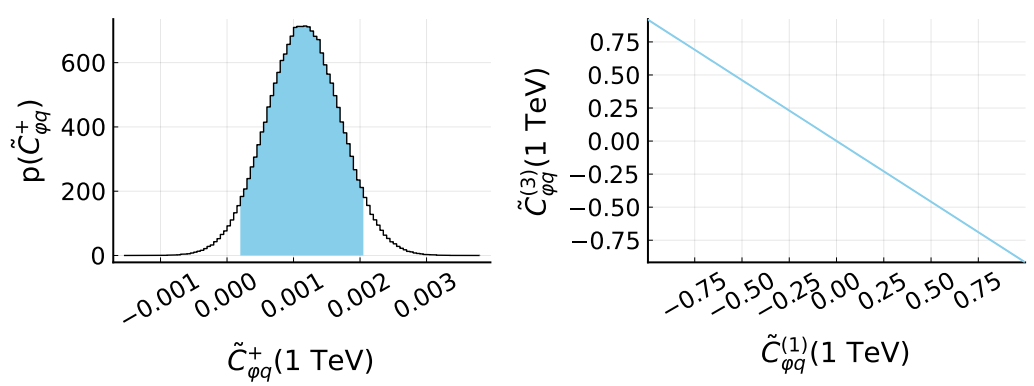

Figure 4. Results of fits to $Z b \bar{b}$ data considering $\tilde{C}_{\varphi q}^{+}$(left) and $\tilde{C}_{\varphi q}^{(1)}$ and $\tilde{C}_{\varphi q}^{(3)}$ (right) as degrees of freedom. Shown are the one-dimensional (left) and two-dimensional (right) projection of the posterior distribution obtained in fits of one and two coefficients, respectively. Colored areas correspond to the smallest intervals containing $90 \%$ of the posterior distribution. For the prior we consider an interval $-1 \leq \tilde{C}_{i} \leq 1$.

\begin{tabular}{|cccccc|}
\hline Process & Observable & $q^{2}$ bin $\left[\mathrm{GeV}^{2}\right]$ & Experiment & Ref. & SM ref. \\
\hline $\bar{B} \rightarrow X_{s} \gamma$ & $\mathrm{BR}_{E_{\gamma}>1.6 \mathrm{GeV}}$ & - & HFLAV & {$[80]$} & {$[94]$} \\
$B^{0} \rightarrow K^{*} \gamma$ & $\mathrm{BR}$ & - & HFLAV & {$[80]$} & {$[65]$} \\
$B^{+} \rightarrow K^{*+} \gamma$ & $\mathrm{BR}$ & - & HFLAV & {$[80]$} & {$[65]$} \\
\hline $\bar{B} \rightarrow X_{s} \ell^{+} \ell^{-}$ & $\mathrm{BR}$ & {$[1,6]$} & BaBar & {$[95]$} & {$[96]$} \\
& $A_{\mathrm{FB}}$ & {$[1,6]$} & Belle & {$[97]$} & {$[96]$} \\
$B_{s} \rightarrow \mu^{+} \mu^{-}$ & $\mathrm{BR}$ & - & LHCb & {$[98]$} & {$[65]$} \\
$B^{0} \rightarrow K^{*} \mu^{+} \mu^{-}$ & $F_{L}, P_{1}, P_{2}, P_{3}$, & {$[1.1,6]$} & LHCb & {$[99]$} & {$[65]$} \\
$B_{4}^{0}, P_{5}^{\prime}, P_{6}^{\prime}, P_{8}^{\prime}$ & & & & & \\
$B^{+} \rightarrow \mu^{+} \mu^{+} \mu^{-}$ & $d \mathrm{BR} / d q^{2}$ & {$[1,6]$} & LHCb & {$[100]$} & {$[65]$} \\
$B^{+} \rightarrow K^{+*} \mu^{+} \mu^{-}$ & $d \mathrm{BR} / d q^{2}$ & {$[1,6]$} & LHCb & {$[100]$} & {$[65]$} \\
$B_{s} \rightarrow \phi \mu^{+} \mu^{-}$ & $F_{L}, S_{3}, S_{4}, S_{7}$ & {$[1,6]$} & LHCb & {$[101]$} & {$[65]$} \\
$\Lambda_{b} \rightarrow \Lambda \mu^{+} \mu^{-}$ & $d \mathrm{BR} / d q^{2}$ & {$[15,20]$} & LHCb & {$[102]$} & {$[65]$} \\
\hline$B_{s}-\bar{B}_{s}$ mixing & $\Delta M_{s}$ & - & HFLAV & {$[80]$} & {$[75]$} \\
\hline
\end{tabular}

Table 3. B-physics measurements included in the fit. For observables measured in $q^{2}$ bins (where $q^{2}$ denotes the squared invariant dilepton mass) we include only one bin due to unknown correlations between different bins for consistency. 

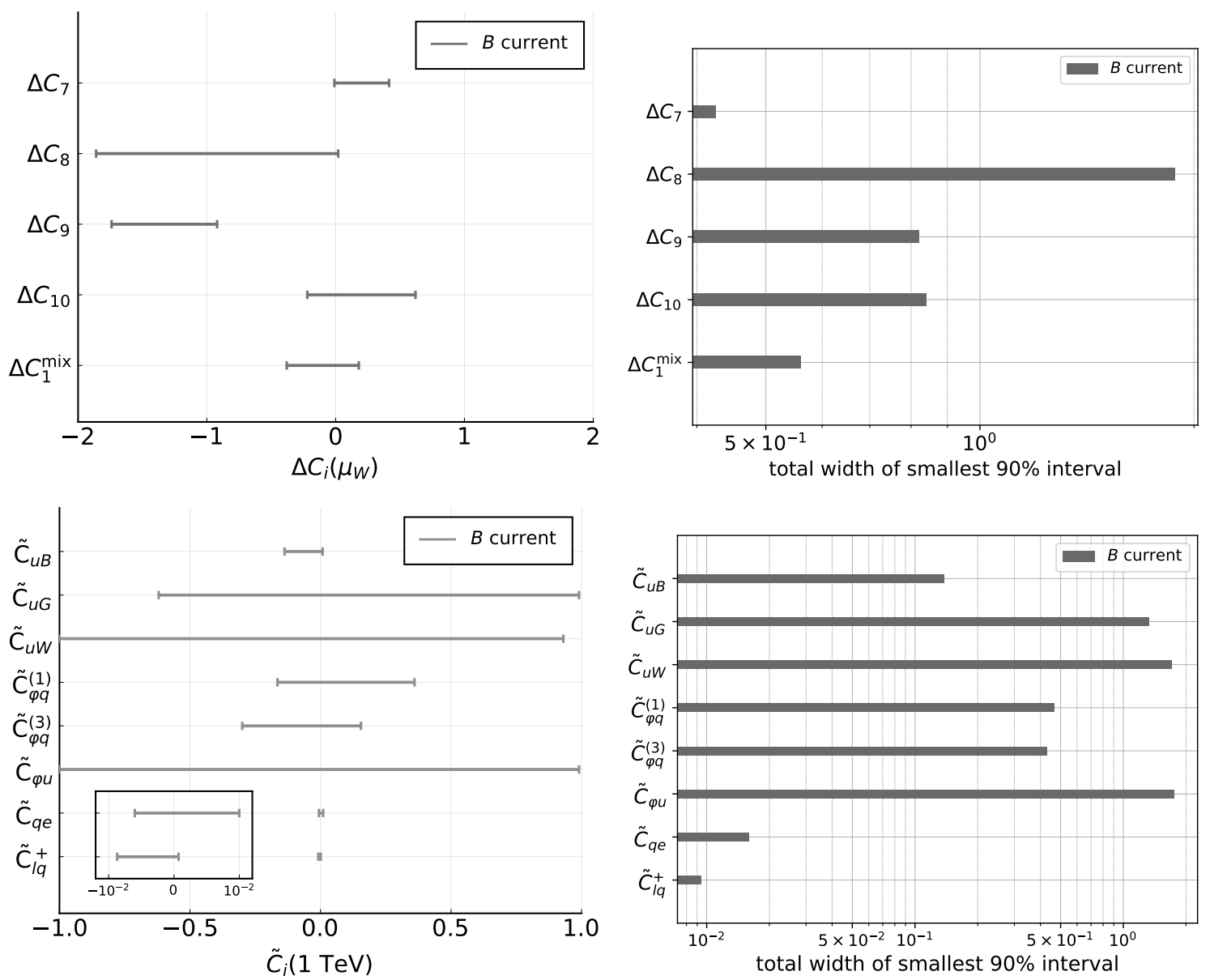

Figure 5. Constraints on WET coefficients $\Delta C_{i}$ at the scale $\mu=\mu_{W}$ (upper plots) and on SMEFT coefficients (lower plots) from measurements of $B$ observables in table 3 . Shown are the marginalized smallest intervals containing $90 \%$ of the posterior probability (left) and the total width of these intervals (right) obtained in a fit of five WET (upper plots) and eight SMEFT coefficients (lower plots) to the data. The fit is performed using a uniform distribution over the interval $-2 \leq \Delta C_{i} \leq 2$ $\left(-1 \leq \tilde{C}_{i} \leq 1\right)$ as a prior for the WET (SMEFT) coefficients.

and exclusive branching ratios of $b \rightarrow s \gamma$ transitions, total and differential branching ratios of various $b \rightarrow s \mu^{+} \mu^{-}$processes, inclusive branching ratios and asymmetries of $b \rightarrow s \ell^{+} \ell^{-}$ transitions, and angular distributions of $B^{0} \rightarrow K^{*} \mu^{+} \mu^{-}$and $B_{s} \rightarrow \phi \mu^{+} \mu^{-}$. In the case of $B_{s} \rightarrow \mu^{+} \mu^{-}$, we consider the latest results presented by the LHCb collaboration [98]. We compute SM predictions and uncertainties with flavio [65]. In addition, we also include the mass difference $\Delta M_{s}$ measured in $B_{s}-\bar{B}_{s}$ mixing, with SM prediction from ref. [75]. Note that we do not take into account measurements of the $B \rightarrow K^{(*)} \nu \bar{\nu}$ branching ratios as only upper limits are presently available by Belle [103] and BaBar [104], which can not be considered in EFTfitter.

In figure 5 (upper plots) we give constraints on BSM contributions $\Delta C_{i}$ to WET coefficients at the scale $\mu_{W}=m_{W}$ from a fit of five coefficients to the data in table 3 . The strongest constraints exist for $\Delta C_{1}^{\text {mix }}$ and $\Delta C_{7}$ for which the width of the smallest $90 \%$ 
interval is about $5.5 \times 10^{-1}$ and $4 \times 10^{-1}$, respectively. The weakest constraints are obtained for $\Delta C_{8}$ as this coefficient contributes via mixing only. For $\Delta C_{9}$ we observe deviations from the SM. This effect stems mainly from measurements of angular distributions of $b \rightarrow s \mu^{+} \mu^{-}$ by $\mathrm{LHCb}$ and is widely known and discussed in literature, see e.g. ref. [105] for a detailed discussion. The exact deviation from the SM depends on the measurements considered in the fit. For the observables in table 3 we find deviations mostly in $\Delta C_{9}$ while $\Delta C_{10}$ is SM like. The constraints on the WET coefficients $\Delta C_{i}$ can be translated into constraints on eight SMEFT coefficients (figure 5, lower plots) discussed in more detail in the next subsection, which are strongly correlated due to the matching conditions, see eqs. (2.12)(2.18). Nevertheless, strong constraints at the level of $\mathcal{O}\left(10^{-2}\right)$ are found for the fourfermion coefficients. Constraints on the remaining coefficients are around one $\left(\tilde{C}_{u B}, \tilde{C}_{\varphi q}^{(1)}\right.$, $\left.\tilde{C}_{\varphi q}^{(3)}\right)$ to two $\left(\tilde{C}_{u G}, \tilde{C}_{u W}, \tilde{C}_{\varphi u}\right)$ orders of magnitude weaker. Note that deviations from the $\mathrm{SM}$, which are present in the one-dimensional projection of the posterior distribution of $\Delta C_{9}$, can not be seen in the one-dimensional results in the SMEFT basis. This is due to the strong correlations among the SMEFT coefficients induced by the matching conditions.

\subsection{Combined fit to current data}

Combining top-quark, $Z b b$, and $B$ observables allows to constrain a larger number of SMEFT coefficients compared to fits using only the individual datasets. Specifically, the coefficients constrained by data in tables 2 and 3 and $Z b b$ data are

$$
\tilde{C}_{u B}, \quad \tilde{C}_{u G}, \quad \tilde{C}_{u W}, \quad \tilde{C}_{\varphi q}^{(1)}, \quad \tilde{C}_{\varphi q}^{(3)}, \quad \tilde{C}_{\varphi u}, \quad \tilde{C}_{q e}, \quad \tilde{C}_{l q}^{+} .
$$

From the fit of these eight coefficients to the combined dataset we obtain the results shown in figure 6 . The strongest constraints are on $\tilde{C}_{q e}$ and $\tilde{C}_{l q}^{+}$, whose width of the smallest interval is around $(5-6) \times 10^{-3}$. This is expected, since both coefficients give sizable contributions to $\Delta C_{9}$ and $\Delta C_{10}$ at tree level (2.12). For $\tilde{C}_{u B}, \tilde{C}_{u G}, \tilde{C}_{u W}, \tilde{C}_{\varphi q}^{(1)}$ and $\tilde{C}_{\varphi q}^{(3)}$ the constraints are about one order of magnitude weaker, with a width of around $(5-7) \times 10^{-2}$. While constraints on $\tilde{C}_{u G}$ and $\tilde{C}_{u W}$ coincide with those derived from fits to top-quark data, the combination of the three datasets significantly tightens constraints on the other three coefficients. For $\tilde{C}_{u B}$ this enhancement stems from different sensitivities of top-quark and $B$ data, as already discovered in ref. [18]. The effect of the different datasets is shown in detail in figure 7 (left), where we give the two-dimensional projection of the posterior distributions obtained in fits to different datasets in the $\tilde{C}_{u B}-\tilde{C}_{u W}$ plane. Here, the effects are even more pronounced compared to ref. [18], since a larger set of $B$ observables is considered here. Constraints on $\tilde{C}_{\varphi q}^{(1)}$ and $\tilde{C}_{\varphi q}^{(3)}$ (figure 6) are tightened by the inclusion of $Z b b$ data, which strongly constraints $\tilde{C}_{\varphi q}^{+}$, as well as the strong constraints on $\tilde{C}_{\varphi q}^{(3)}$, which arise from the combination of top-quark and $B$ physics data (see figure 7 ). As can be seen, in the combined fit the SM is included in the smallest intervals containing $90 \%$ of the posterior distribution of $\tilde{C}_{\varphi q}^{(1)}$ and $\tilde{C}_{\varphi q}^{(3)}$, which is shown in detail in figure 13. The weakest constraints are found for $\tilde{C}_{\varphi u}$, since contributions to $B$ physics data are strongly suppressed, and $t \bar{t} Z$ production offers only a limited sensitivity, as we can already see in figure 3. 

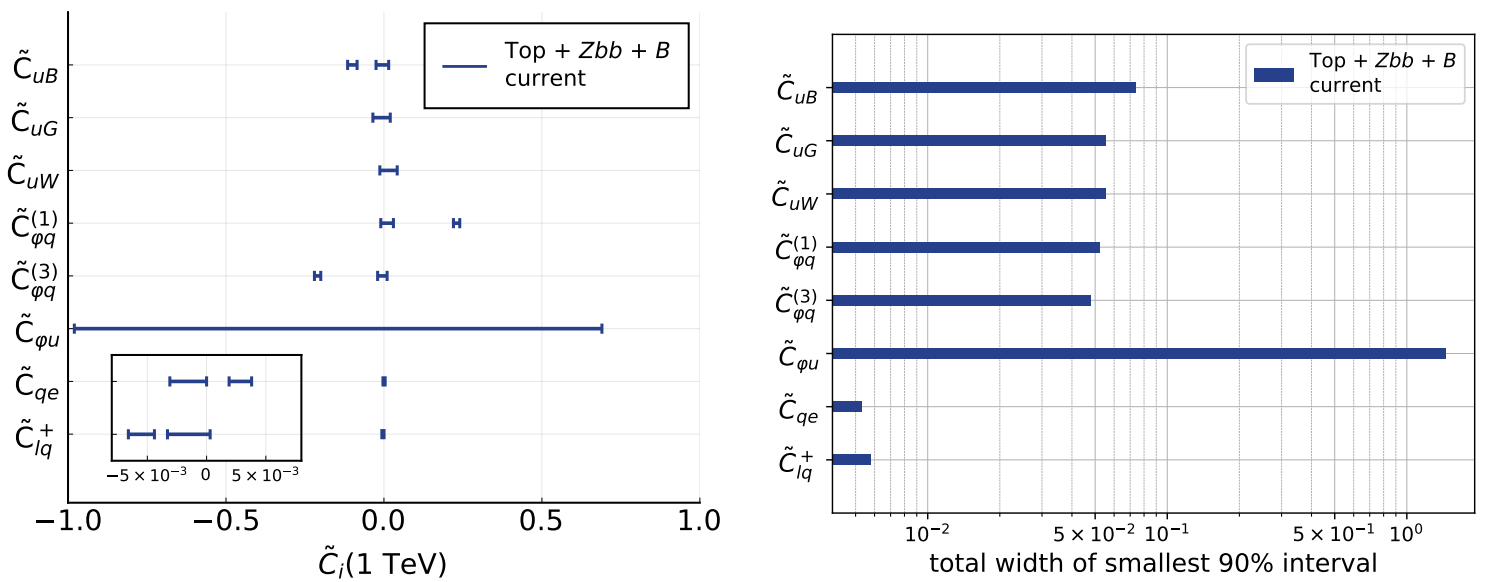

Figure 6. Constraints on SMEFT coefficients $\tilde{C}_{i}$ in eq. (4.3) from a fit of eight coefficients to topquark data in table $2, Z b b$ data, and $B$ physics data in table 3 . Shown are the smallest intervals containing $90 \%$ posterior probability (left) and the total width of these intervals (right). For the prior we assume a uniform distribution over the interval $-1 \leq \tilde{C}_{i} \leq 1$.
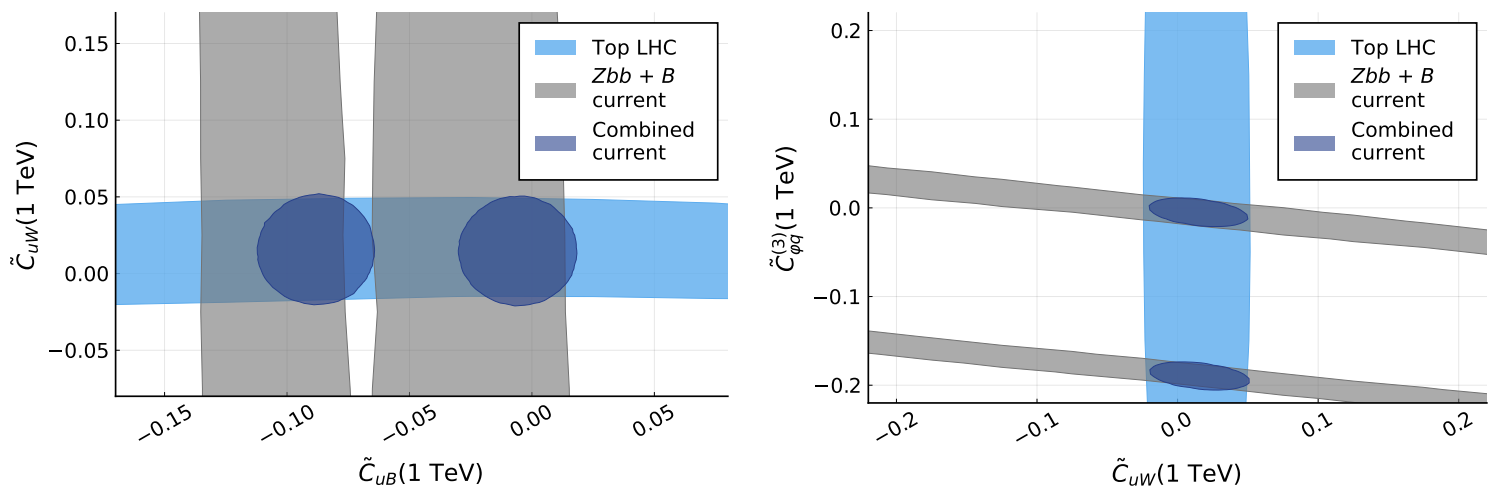

Figure 7. Examples for two-dimensional posterior distributions of SMEFT coefficients $\tilde{C}_{i}$ in eq. (4.3) obtained in a fit of eight coefficients to top-quark data (light blue), $B$ physics data (grey) and the combined dataset including $Z b b$ data (blue). Shown are the smallest intervals containing $90 \%$ of the posterior distribution. For the prior we assume a uniform distribution over the interval $-1 \leq \tilde{C}_{i} \leq 1$.

Interestingly, we find two solutions for several coefficients; one of which is SM like, while the other one deviates from the SM: $\tilde{C}_{u B}, \tilde{C}_{\varphi q}^{(1)}, \tilde{C}_{\varphi q}^{(3)}$, and the four-fermion coefficients $\tilde{C}_{q e}$ and $\tilde{C}_{l q}^{+}$. As can be seen in figure 7 , the second solutions stem from the correlations between the coefficients introduced by matching the SMEFT basis onto the WET basis. Since the number of degrees of freedom is smaller in WET, correlations among the coefficients arise. Inclusion of top-quark data reduces these correlations, however, for the five coefficients the sensitivity of top-quark observables does not suffice to exclude the non-SM branches completely given present data and theory predictions. Without further input this ambiguity cannot be resolved.

We compare our results to those reported in a recent study on $b \rightarrow s \ell^{+} \ell^{-}$transitions [106]. In contrast to our analysis, operators are defined in a basis of diagonal down- 
type quark Yukawa couplings, which leads to an additional factor of $1 /\left(V_{t b} V_{t s}^{*}\right)$. Taking this factor into account, the results from [106] correspond to $\tilde{C}_{l q}^{+}, \tilde{C}_{q e} \sim 10^{-3}$, consistent with figure 6 . Repeating our fit with $\tilde{C}_{q e}$ and $\tilde{C}_{l q}^{+}$only, we find agreement with ref. [106].

We also comment on Drell-Yan production at the LHC. Amongst the couplings with top-quark focus considered in this works, $(2.8)$, this concerns $\tilde{C}_{\varphi q}^{+}, \tilde{C}_{q e}$ and $\tilde{C}_{l q}^{+}$, just like $b \rightarrow$ $s \ell^{+} \ell^{-}$and $Z \rightarrow b \bar{b}$. Drell-Yan limits from existing data and a $3000 \mathrm{fb}^{-1}$ future projection for the semileptonic four-fermion operators with $b$-quarks are at the level of $\mathcal{O}\left(10^{-2}\right)[107,108]$, and weaker than in the combined fit, figure 6 . Note, with the flavor of the initial quarks in $p p$-collisions undetermined an actual measurement of a quark flavor-specific coefficient is not possible. A detailed study of the implications of Drell-Yan processes for a global fit is beyond the scope of this work.

\section{$5 \quad$ Impact of future colliders}

Both the HL-LHC operating at $14 \mathrm{TeV}$ with an integrated luminosity of $3000 \mathrm{fb}^{-1}$ [42] and Belle II at $50 \mathrm{ab}^{-1}$ [43] are going to test the SM at the next level of precision. In section 5.1, we work out the impact of future measurements at these facilities on the SMEFT Wilson coefficients.

A first study of top-quark physics at the proposed lepton collider CLIC has been provided in ref. [27]. CLIC is intended to operate at three different center-of-mass energies: $380 \mathrm{GeV}, 1.4 \mathrm{TeV}$, and $3 \mathrm{TeV}$ and two different beam polarizations are foreseen by the accelerator design: a longitudinal polarization of $\pm 80 \%$ for the electron beam and no polarization of the positron beam. We investigate the impact of measurements with the currently foreseen precision of such a lepton collider on the constraints of SMEFT Wilson coefficients in section 5.2.

We combine existing data with HL-LHC, Belle II and CLIC projections in section 5.3.

\subsection{Expected constraints from HL-LHC and Belle II}

For the expected experimental uncertainties at the HL-LHC and Belle II we adopt estimates of the expected precision by ATLAS, CMS and Belle II collaborations [42, 43, 109-111]. If no value for the systematic uncertainties is given, we assume that these uncertainties shrink by a factor of two compared to the current best measurement, which is the case for the $t \bar{t}$ and $t \bar{t} Z$ cross sections, the $W$ boson helicity fractions, and the top-quark decay width. In addition, we make the assumption that theory uncertainties shrink by a factor of two compared to the current SM uncertainties due to improved MC predictions and higher-order calculations. We summarize the observables and references for the expected experimental and theory precision at HL-LHC and Belle II in table 4. For the purpose of the fit, we consider present central values of measurements for the future projections. If no measurement is available, we consider the SM for central values.

For fiducial cross sections of $t \bar{t} \gamma$ production, an analysis with the expected uncertainties is provided in refs. [42, 109]. For both the dilepton and single-lepton cross section we consider the precision of the channel with the largest experimental uncertainty as our estimate. For $t \bar{t} Z$ production we follow the analysis in refs. [42, 110] and scale statistical 


\begin{tabular}{|cccccc|}
\hline Process & Observable & $q^{2}$ bin $\left[\mathrm{GeV}^{2}\right]$ & Experiment & Ref. & SM ref. \\
\hline \multicolumn{1}{c}{$t \bar{t} \gamma$} & $\sigma^{\text {fid }}(t \bar{t} \gamma, 1 \ell), \sigma^{\text {fid }}(t \bar{t} \gamma, 2 \ell)$ & - & ATLAS & {$[42,109]$} & {$[81,82]$} \\
$t \bar{t} Z$ & $\sigma^{\text {inc }}(t \bar{t} Z)$ & - & CMS & {$[42,110]$} & {$[84-86]$} \\
\hline$t \bar{t}$ & $\sigma^{\text {inc }}(t \bar{t})$ & - & CMS & {$[42,111]$} & {$[88]$} \\
& $F_{0}, F_{L}$ & - & - & - & {$[90]$} \\
& $\Gamma_{t}$ & - & - & - & {$[92]$} \\
\hline $\bar{B} \rightarrow X_{s} \gamma$ & $\mathrm{BR}_{E_{\gamma}>1.6 \mathrm{GeV}}$ & - & Belle II & {$[43]$} & {$[94]$} \\
$B^{0} \rightarrow K^{*} \gamma$ & $\mathrm{BR}$ & - & Belle II & {$[43]$} & {$[65]$} \\
$B^{+} \rightarrow K^{+*} \gamma$ & $\mathrm{BR}$ & - & Belle II & {$[43]$} & {$[65]$} \\
\hline $\bar{B} \rightarrow X_{s} \ell^{+} \ell^{-}$ & $\mathrm{BR}, A_{\mathrm{FB}}$ & {$[3.5,6]$} & Belle II & {$[43]$} & {$[96]$} \\
$B^{0} \rightarrow K^{*} \mu^{+} \mu^{-}$ & $F_{L}, P_{1}, P_{2}, P_{3}$, & {$[1.1,2.5],[2.5,4],[4,6]$} & Belle II & {$[43]$} & {$[65]$} \\
\hline$B^{0} \rightarrow P_{5}^{\prime}, P_{6}^{\prime}, P_{8}^{\prime}$ & $\mathrm{BR}$ & - & Belle II & {$[43]$} & {$[65]$} \\
\hline
\end{tabular}

Table 4. Overview of observables considered at future HL-LHC and Belle II projections. For each process we denote the references for the experimental projection and the SM prediction. In case of the $[1.1,2.5] q^{2}$ bin for $B^{0} \rightarrow K^{*} \mu^{+} \mu^{-}$we consider the Belle II projection of the [1.0, 2.5] bin for the expected experimental uncertainties.

uncertainties according to the luminosity. For systematic uncertainties we assume for simplicity a reduction by a factor of 2 . For estimating the expected precision of the total $t \bar{t}$ production cross section, we base our assumptions on the study of differential $t \bar{t}$ cross sections in refs. [42, 111]. For the uncertainties we apply the same assumptions as for $t \bar{t} Z$. As the $W$ boson helicity fractions and the top-quark decay width are not discussed in ref. [42], we treat them in the same way as the $t \bar{t}$ cross section for simplicity.

For measurements of $b \rightarrow s$ transitions we take the estimates in ref. [43] into account. For the $b \rightarrow s \gamma$ inclusive branching ratio we take the precision for the $\operatorname{BR}\left(\bar{B} \rightarrow X_{s} \gamma\right)_{E_{\gamma}>1.9 \mathrm{GeV}}$ measurement and assume that the same uncertainties apply for $E_{\gamma}>1.6 \mathrm{GeV}$. In case of $B^{(+)} \rightarrow K^{(+) *} \gamma$, we directly include the estimated precision in ref. [43]. Similarly, for the inclusive decay $\bar{B} \rightarrow X_{s} \ell^{+} \ell^{-}$we use the expected precision for the $3.5 \mathrm{GeV}^{2} \leq q^{2} \leq 6 \mathrm{GeV}^{2}$ bin. We also considered other bins for this observable and found very comparable sensitivity. Finally, for $B \rightarrow K^{*} \mu^{+} \mu^{-}$we include the angular distribution observable $P_{i}^{(\prime)}$ in different $q^{2}$ bins, and study the implications of the anomalies found in present data of $b \rightarrow s \mu^{+} \mu^{-}$angular distributions.

Combining top-quark and $B$ observables at HL-LHC and Belle II allows to test a total of nine SMEFT coefficients, see figure 8. In order to derive these constraints with EFTfitter, we have chosen a smaller prior $\left|\tilde{C}_{i}\right| \leq 0.1$ for the four-fermion coefficients because the posterior distribution lies only in a very small region, and a larger prior would lead to convergence issues. At this point, we neglect subleading contributions from $\tilde{C}_{e u}$ and $\tilde{C}_{l u}$, which are considered in section 5.3. As can be seen, the observables strongly constrain all coefficients except for $\tilde{C}_{\varphi u}$, which is only very weakly constrained, $\mathcal{O}(1)$, due to the low sensitivity in both $t \bar{t} Z$ and $B$ observables. Conversely, the strongest constraints 

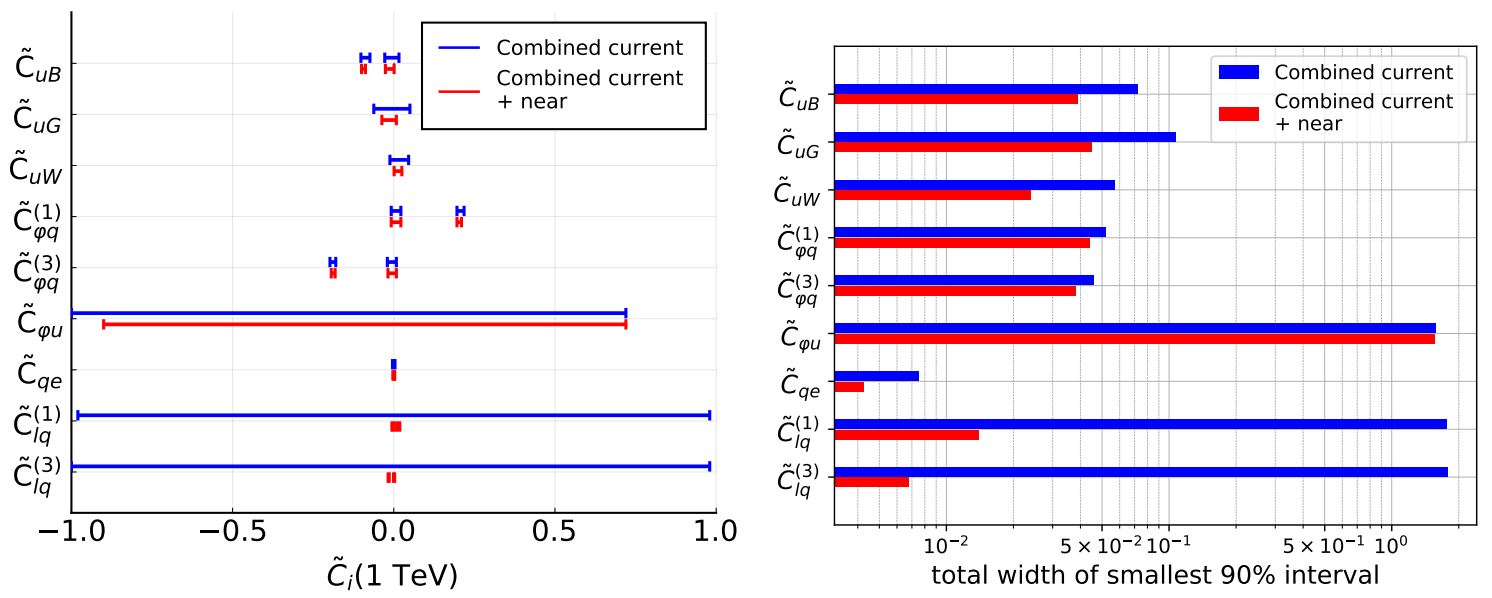

Figure 8. Constraints on coefficients $\tilde{C}_{i}$ from fits of nine coefficients to current top-quark and $B$ measurements in tables 2 and 3 (blue) and to current measurments and projections of top-quark and $B$ observables in tables 2-4 (red). Shown are the marginalized smallest intervals containing $90 \%$ posterior probability (left) and the total widths of these intervals (right).

are found for the four-fermion coefficients, around $4 \times 10^{-3}$ and $(7-15) \times 10^{-3}$ for $\tilde{C}_{q e}$ and $\tilde{C}_{l q}^{(1,3)}$, respectively. The inclusion of $b \rightarrow s \nu \bar{\nu}$ observables allows to test $\tilde{C}_{l q}^{(1)}$ and $\tilde{C}_{l q}^{(3)}$ independently due to the orthogonal sensitivity compared to $b \rightarrow s \ell^{+} \ell^{-}$observables, as indicated in figure 9. We observe that the interval obtained in the combined fit is significantly smaller than expected from the simple overlay of constraints from $b \rightarrow s \nu \bar{\nu}$ and $b \rightarrow s \ell^{+} \ell^{-}$observables. The reason is, that the posterior distribution is constrained in the multi-dimensional hyperspace, and the combination significantly reduces correlations among different coefficients. In addition, we find that two solutions for $\tilde{C}_{l q}^{(1)}$ and $\tilde{C}_{l q}^{(3)}$ are allowed: one is close to the SM, while the other is around $\tilde{C}_{l q}^{(1)} \sim-\tilde{C}_{l q}^{(3)} \sim 10^{-3}$, and deviates strongly from the SM. Without further input, this ambiguity can not be resolved. Constraints on the remaining coefficients $\tilde{C}_{u B}, \tilde{C}_{u G}, \tilde{C}_{u W}, \tilde{C}_{\varphi q}^{(1)}$, and $\tilde{C}_{\varphi q}^{(3)}$ are in the range $(2-5) \times 10^{-2}$. Here, the higher precision in the near-future scenario tightens constraints on $\tilde{C}_{u B}(t \bar{t} \gamma$ and $b \rightarrow s \gamma), \tilde{C}_{u G}(t \bar{t})$, and $\tilde{C}_{u W}$ (helicity fractions) by a factor of around 2 , while constraints on $\tilde{C}_{\varphi q}^{(1,3)}$ remain mostly unchanged. Note that the inclusion of additional measurements in the near-future projection does not suffice to resolve the second solutions observed for several coefficients.

\subsection{CLIC projections}

In table 5 we list the top-quark observables for the CLIC future projections considered in this work. This set comprises total cross sections of $t \bar{t}$ production and forward-backward asymmetries $A_{\mathrm{FB}}$ as observables for different energy stages and beam polarizations [27]. We use the current SM predictions as nominal values, which include NLO QCD corrections [40].

In figure 10 we give the results for a fit to the CLIC projections in table 5. A smaller prior $\left|\tilde{C}_{i}\right| \leq 0.1$ is employed for the four-fermion coefficients due to the small size of the posterior distribution. We explicitly checked that we do not remove any solutions. 


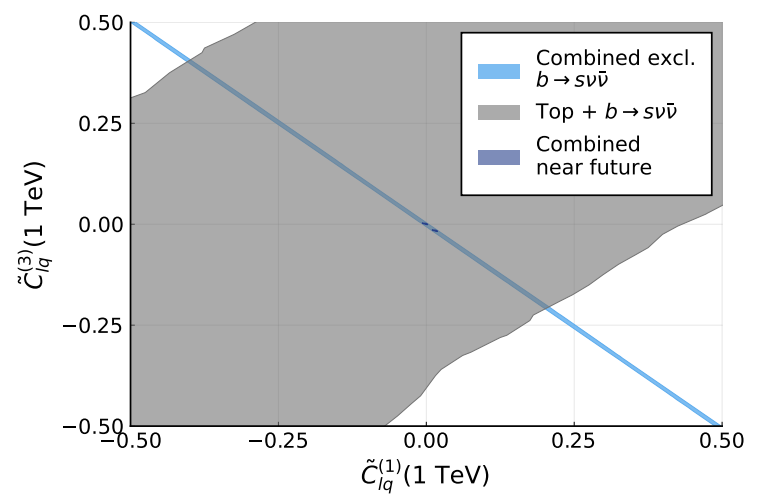

Figure 9. Two-dimensional projection of the posterior distribution in the $\tilde{C}_{l q}^{(1)}-\tilde{C}_{l q}^{(3)}$ plane. Shown are smallest intervals containing $90 \%$ of the posterior distribution obtained in fits of nine coefficients in the near future projection combining top-quark observables with all beauty observables except $b \rightarrow s \nu \bar{\nu}$ ones (light blue), only with $b \rightarrow s \nu \bar{\nu}$ ones (grey), and the combined set (dark blue).

\begin{tabular}{|ccccc|}
\hline Observable & $\sqrt{s}$ & Polarization $\left(e^{-}, e^{+}\right)$ & Ref. experiment & SM ref. \\
\hline$\sigma_{t \bar{t}}, A_{\mathrm{FB}}$ & $380 \mathrm{GeV}$ & $( \pm 80 \%, 0)$ & {$[27]$} & {$[40]$} \\
$\sigma_{t \bar{t}}, A_{\mathrm{FB}}$ & $1.4 \mathrm{TeV}$ & $( \pm 80 \%, 0)$ & {$[27]$} & {$[40]$} \\
$\sigma_{t \bar{t}}, A_{\mathrm{FB}}$ & $3 \mathrm{TeV}$ & $( \pm 80 \%, 0)$ & {$[27]$} & {$[40]$} \\
\hline
\end{tabular}

Table 5. Observables at different energies and polarizations for $t \bar{t}$ production at CLIC [27]. SM predictions are taken from [40].

Constraints on $\tilde{C}_{u G}$, which contributes via mixing only, are at the level of $4 \times 10^{-1}$, and weaker compared to the ones on the remaining Wilson coefficients. For $\tilde{C}_{\varphi q}^{-}$and $\tilde{C}_{\varphi u}$ the width of the smallest $90 \%$ interval is at the level of $10^{-1}$. In comparison, constraints on $\tilde{C}_{u B}$ and $\tilde{C}_{u W}$ are found to be stronger by one order of magnitude. Even tighter constraints are obtained for four-fermion interactions, where the width of the smallest interval is at the level of $(2-6) \times 10^{-4}$. Interestingly, while figure 10 shows results of a fit treating $\tilde{C}_{l q}^{-}$as a degree of freedom, the inclusion of RGE effects on $\tilde{C}_{l q}^{(1)}$ and $\tilde{C}_{l q}^{(3)}$ allows to distinguish both coefficients. The reason is that they develop differently under the RGE flow where corrections are at the level of $\mathcal{O}(1) \%$. Thus, both coefficients can be constrained simultaneously to a level of $\mathcal{O}\left(10^{-2}\right)$, as shown in detail in figure 11.

\subsection{Combined fit}

Combining measurements and near-future projections of top-quark physics and $B$ physics in tables 2-4 with the projections for top-quark observables at a CLIC-like lepton collider allows to constrain all eleven SMEFT coefficients considered in this analysis.

In figure 11 we give results from fits of all eleven coefficients to current data (tables 2, 3, and $Z b \bar{b}$ data) and near-future projections (table 4) (light blue), to CLIC projections for top-quark observables (table 5) (grey) and the combined set (blue). It can be observed that the fit to the combined set of observables allows to constrain all eleven SMEFT Wilson 

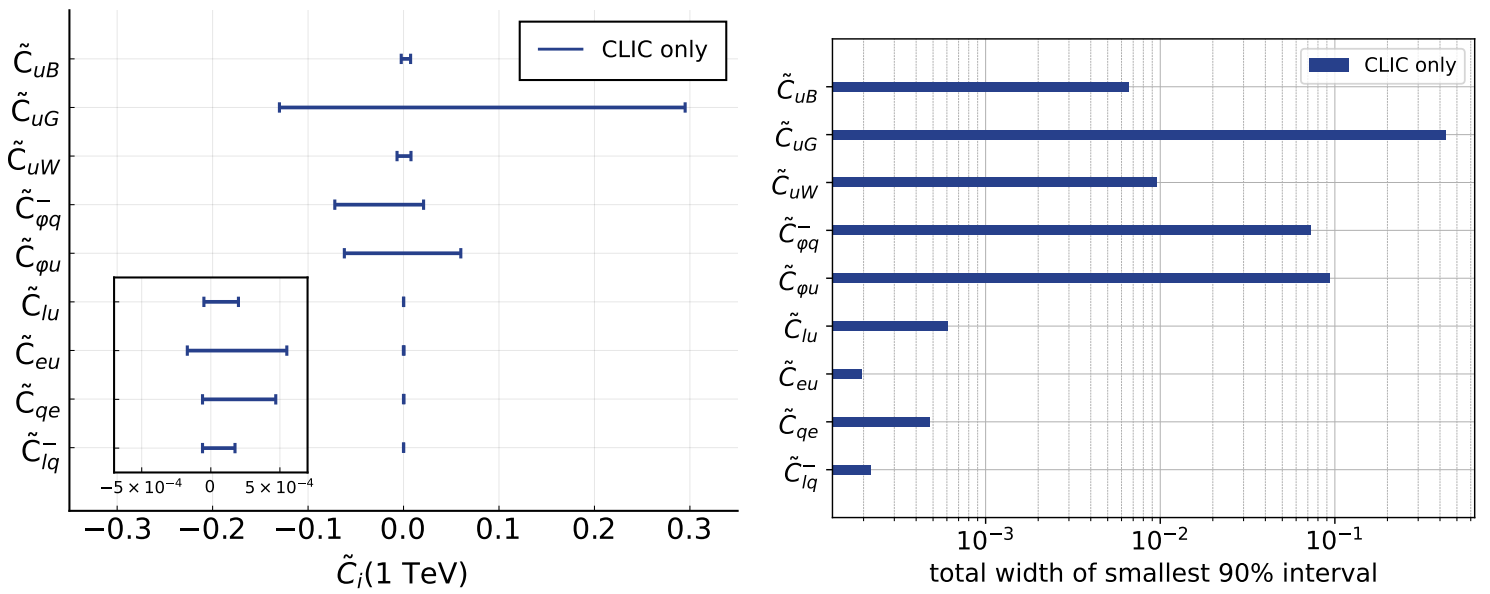

Figure 10. Constraints on coefficients $\tilde{C}_{i}$ from a fit of nine coefficients to CLIC observables in table 5. Shown are the marginalized smallest intervals containing $90 \%$ posterior probability (left) and the total widths of these intervals (right).
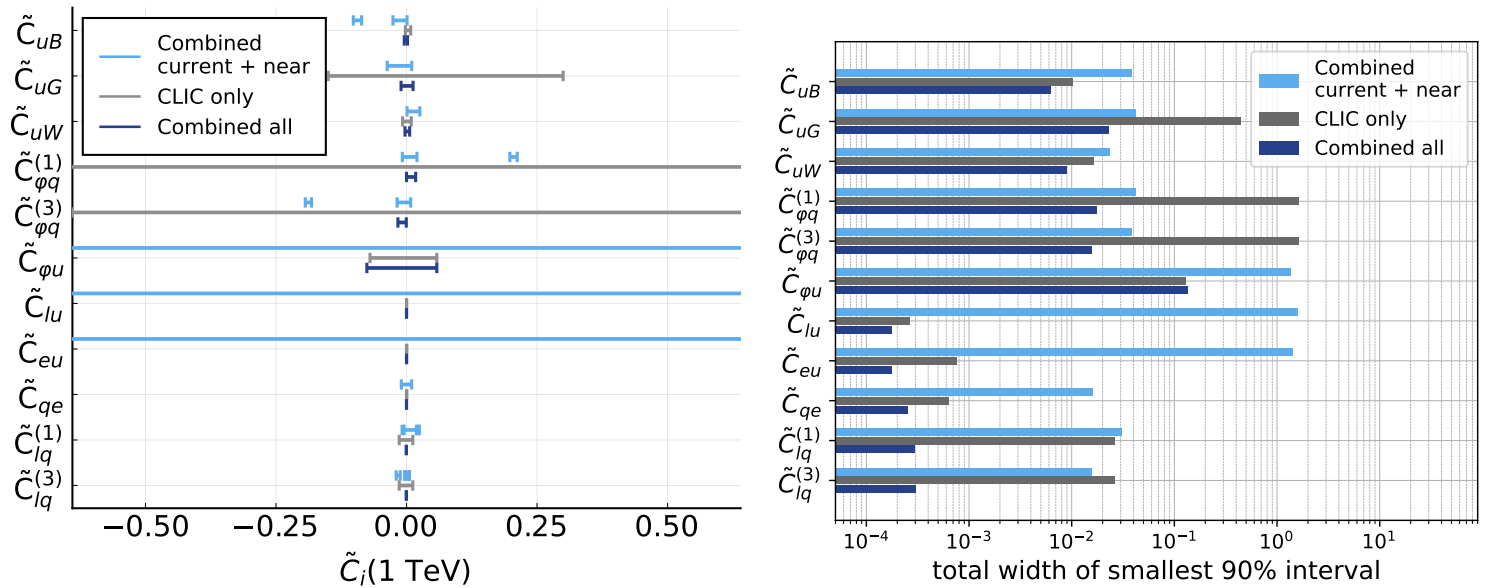

Figure 11. Constraints on coefficients $\tilde{C}_{i}$ obtained fitting eleven coefficients to top-quark and $B$ data and near-future projections at HL-LHC and Belle II in tables 2-4 and CLIC future projections in table 5. Shown are the marginalized smallest intervals containing $90 \%$ posterior probability (left) and the total widths of these intervals (right).

coefficients. Flat directions in the parameter space of the coefficients are removed in the global fit. The strongest constraints are obtained for the four-fermion operators and are at the level of $\mathcal{O}\left(10^{-4}\right)$. Constraints on the other operators are weaker and at the level of $\mathcal{O}\left(10^{-1}\right)$ for $\tilde{C}_{\varphi u}$ and $\mathcal{O}\left(10^{-2}\right)$ for the remaining coefficients.

As learned previously, combining different sets of observables yields stronger constraints on all coefficients. In the case of $\tilde{C}_{\varphi q}^{(1)}$ and $\tilde{C}_{\varphi q}^{(3)}$, which are already strongly constrained by present data and Belle II and HL-LHC projections, additional constrains derived from CLIC projections are orthogonal to those from the remaining observables, see figure 12 (left). This tightens the constraints by a factor of two and allows to exclude the second solution. Similarly, the second solution for $\tilde{C}_{u B}$ still present in the near-future 

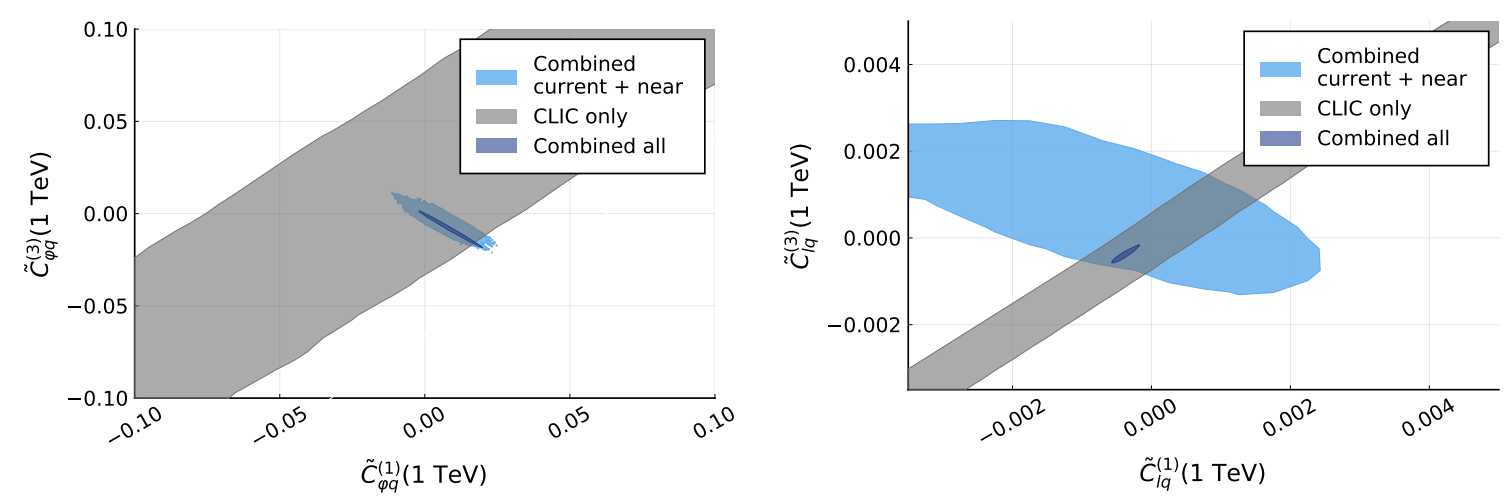

Figure 12. Examples for two-dimensional marginalized posterior distributions of SMEFT coefficients $\tilde{C}_{i}$ obtained in a fit of all eleven coefficients to top-quark and $B$ data in tables 2-4 (light blue), top-quark observables at CLIC in table 5 (grey) and the combined set (blue). Shown are the smallest regions containing $90 \%$ posterior probability. Constraints from the fit on the combined set are so strong that the smallest $90 \%$ region is barely visible in the $\tilde{C}_{l q}^{(1)}-C_{l q}^{(3)}$ plane (plot to the right).

scenario is removed as well. The improvement is particularly significant for $\tilde{C}_{l q}^{(1)}$ and $\tilde{C}_{l q}^{(3)}$. While $b \rightarrow s \ell^{+} \ell^{-}$and $b \rightarrow s \nu \bar{\nu}$ observables allow to test both coefficients simultaneously, the inclusion of CLIC observables is mandatory to remove the second solution, see figure 12 (right). Correlations, which are induced by CLIC observables, between both coefficients are still present, and sizable deviations from the SM can be found, which is shown in more detail in figure 14 in appendix F. These deviations stem from the assumption that Belle II confirms present LHCb data. Interestingly, even though CLIC observables strongly constrain $\tilde{C}_{l q}^{-}$(assuming that the $\mathrm{SM}$ value is measured), the exact position of the smallest $90 \%$ interval on the $\tilde{C}_{\ell q}^{(1)} \sim-\tilde{C}_{\ell q}^{(3)}$ subspace is determined by Belle II results (figure 14). A scenario, in which we assume SM values for Belle II observables, is shown in figure 15 in appendix F, and we find agreement with the SM in this case. While indeed constraints from CLIC projections and top-quark and $B$ data and projections in the near-future scenario have a different sensitivity, the $90 \%$ region for $\tilde{C}_{l q}^{(1)}$ and $\tilde{C}_{l q}^{(3)}$ is significantly smaller than expected by simply overlaying the constraints obtained in fits to the two individual datasets. The reason is that constraints are combined in the full eleven-dimensional hyperspace, and figure 12 only shows two-dimensional projections.

As anticipated in section 2.2 the full, global fit results including CLIC projections are obtained assuming lepton-flavor universality. While in BSM scenarios where lepton generations couple differently the results cannot be applied directly, the findings on the orthogonality of the constraints and synergies between top and beauty continue to hold.

\section{Conclusions}

We performed fits within SMEFT to top-quark pair production, decay, $Z \rightarrow b \bar{b}$ transitions, and $b \rightarrow s$ transitions. We highlight how each of the individual datasets constrains different sets of Wilson coefficients of dimension-six operators affecting top-quark physics at present 
and future colliders. Extending previous works [18], we put an emphasis on semileptonic four-fermion operators, which are of high interest as they may be anomalous according to current flavor data and moreover are essentially unconstrained for top quarks. SU $(2)_{L}$ invariance leads to relations between up-type and down-type quark observables, a wellknown feature with recent applications in semileptonic processes within SMEFT [52]. Here, we exploit this symmetry link between top and beauty observabes at the LHC and a future lepton collider.

Using existing data in tables 2 and 3 as well as $Z b b$ data we constrain eight SMEFT Wilson coefficients with results shown in figure 6. Combining complementary constraints significantly improves the fits compared to using individual datasets alone, see figure 7 . Going beyond existing data, we entertain a near-future scenario with measurements from Belle II and the HL-LHC, and one with an additional lepton collider. While measurements of top-quark observables at the HL-LHC allow to put stronger constraints on the same set of coefficients already tested by present top-quark measurements, a notable qualitative improvement in the near future is the expected observation of $b \rightarrow s \nu \bar{\nu}$ transitions at Belle II, which together with lepton universality allows to probe four-fermion operators in new ways: orthogonal to $b \rightarrow s \ell^{+} \ell^{-}$and very similar as in contact interactions of leptons and top quarks, see figure 1 . Thus, in this near-future scenario a combined fit would allow to probe nine SMEFT coefficients with estimated precision shown in figure 8. Combining the present data and projections for near-future experiments together with projections for a CLIC-like lepton collider, a combined fit enables to constrain the eleven SMEFT coefficients considered in this work, see eq. (2.8), as shown in figure 11. The second solution for $\tilde{C}_{l q}^{(1)}$ and $\tilde{C}_{l q}^{(3)}$ present in fits in the near-future scenario, see figure 8 , could be removed by lepton collider measurements, as demonstrated in figure 12. We stress that a lepton collider allows to probe the coefficients $\tilde{C}_{\varphi u}, \tilde{C}_{l u} \tilde{C}_{e u}$, which would otherwise remain loosely constrained in the fit. In the combined fit, constraints on four-fermion coefficients are obtained at the level of $\mathcal{O}\left(10^{-4}\right)$.

To conclude, in order to extract the strongest constraints on SMEFT coefficients from a global fit of the SMEFT top-quark sector and of relevance to the $b$-anomalies, different collider setups as well as $\mathrm{SU}(2)_{L}$ relations have to be employed to remove flat directions and to test all possible sources of BSM contributions simultaneously. The present study clearly demonstrates the strong new physics impact of a future lepton collider.

Note added. During the finalization of this project a preprint appeared by CMS in which SMEFT coefficients are constrained by top production in association with leptons at the $\sqrt{s}=13 \mathrm{TeV}$ LHC with $41.5 \mathrm{fb}^{-1}$ [112]. The constraints on four-fermion coefficients $\tilde{C}_{q e}$ and $\tilde{C}_{l q}^{-}$are more than one order of magnitude weaker compared to ours using current data, figure 6 . However, the CMS-analysis is sensitive to $\tilde{C}_{e u}, \tilde{C}_{l u}$, otherwise unconstrained by present data. A study of the future physics potential of this type of analysis would be desirable, however, requires detector-level simulations and is beyond the scope of this work. 


\section{Acknowledgments}

We thank Danny van Dyk and Susanne Westhoff for useful discussions on RGE evolution in SMEFT and Christoph Bobeth for comments on matching SMEFT and WET. C.G. is supported by the doctoral scholarship program of the Studienstiftung des deutschen Volkes.

\section{A Weak effective theory}

At energies below the scale $\mu_{W} \sim m_{W}$, physical processes are described by the Weak Effective Theory (WET). All BSM particles which are heavier than $m_{W}$ as well as the top quark and the $W, Z$ and Higgs bosons are integrated out. Both $b \rightarrow s \ell^{+} \ell^{-}$and $b \rightarrow s \gamma$ transitions are described by the following Lagrangian:

$$
\mathcal{L}_{\mathrm{WET}}^{b s}=\frac{4 G_{F}}{\sqrt{2}} V_{t s}^{*} V_{t b} \sum_{i=1}^{10} C_{i}(\mu) Q_{i}(\mu) .
$$

Here, $G_{F}$ is the Fermi-constant, $C_{i}$ are Wilson coefficients and $Q_{i}$ are the corresponding effective operators which are defined as follows:

$$
\begin{aligned}
Q_{1} & =\left(\bar{s}_{L} \gamma_{\mu} T^{a} c_{L}\right)\left(\bar{c}_{L} \gamma^{\mu} T^{a} b_{L}\right), & Q_{2} & =\left(\bar{s}_{L} \gamma_{\mu} c_{L}\right)\left(\bar{c}_{L} \gamma^{\mu} b_{L}\right), \\
Q_{3} & =\left(\bar{s}_{L} \gamma_{\mu} b_{L}\right) \sum_{q}\left(\bar{q} \gamma^{\mu} q\right), & Q_{4} & =\left(\bar{s}_{L} \gamma_{\mu} T^{a} b_{L}\right) \sum_{q}\left(\bar{q} \gamma^{\mu} T^{a} q\right), \\
Q_{5} & =\left(\bar{s}_{L} \gamma_{\mu} \gamma_{\nu} \gamma_{\sigma} b_{L}\right) \sum_{q}\left(\bar{q} \gamma^{\mu} \gamma^{\nu} \gamma^{\sigma} q\right), & Q_{6} & =\left(\bar{s}_{L} \gamma_{\mu} \gamma_{\nu} \gamma_{\sigma} T^{a} b_{L}\right) \sum_{q}\left(\bar{q} \gamma^{\mu} \gamma^{\nu} \gamma^{\sigma} T^{a} q\right), \\
Q_{7} & =\frac{e}{16 \pi^{2}} m_{b}\left(\bar{s}_{L} \sigma^{\mu \nu} b_{R}\right) F_{\mu \nu}, & Q_{8} & =\frac{g_{s}}{16 \pi^{2}} m_{b}\left(\bar{s}_{L} \sigma^{\mu \nu} T^{a} b_{R}\right) G_{\mu \nu}^{a}, \\
Q_{9}^{i j} & =\frac{e^{2}}{16 \pi^{2}}\left(\bar{s}_{L} \gamma_{\mu} b_{L}\right)\left(\bar{\ell}^{i} \gamma^{\mu} \ell^{j}\right), & Q_{10}^{i j} & =\frac{e^{2}}{16 \pi^{2}}\left(\bar{s}_{L} \gamma_{\mu} b_{L}\right)\left(\bar{\ell}^{i} \gamma^{\mu} \gamma_{5} \ell^{j}\right),
\end{aligned}
$$

with chiral left (right) projectors $L(R)$ and the field strength tensor of the photon $F_{\mu \nu}$. We denote charged leptons with $\ell$ and neglect contributions proportional to the subleading CKM-matrix element $V_{u b}$ and to the strange-quark mass.

The effective Lagrangian for $b \rightarrow s \bar{\nu} \nu$ transitions can be written as

$$
\mathcal{L}_{\mathrm{WET}}^{\nu}=\frac{4 G_{F}}{\sqrt{2}} V_{t s}^{*} V_{t b} \sum_{i, j=1}^{3}\left(C_{L}^{i j}(\mu) Q_{L}^{i j}(\mu)+C_{R}^{i j}(\mu) Q_{R}^{i j}(\mu)\right),
$$

with effective operators

$$
Q_{L}^{i j}=\frac{e^{2}}{16 \pi^{2}}\left(\bar{s}_{L} \gamma_{\mu} b_{L}\right)\left(\bar{\nu}^{i} \gamma^{\mu}\left(1-\gamma_{5}\right) \nu^{j}\right), \quad Q_{R}^{i j}=\frac{e^{2}}{16 \pi^{2}}\left(\bar{s}_{R} \gamma_{\mu} b_{R}\right)\left(\bar{\nu}^{i} \gamma^{\mu}\left(1-\gamma_{5}\right) \nu^{j}\right) .
$$

Assuming flavor universality, only diagonal terms $i=j$ contribute, and all three flavors couple with identical strength. The $B_{s}-\bar{B}_{s}$ mass difference $\Delta M_{s}$ can be described as

$$
\mathcal{L}_{\mathrm{WET}}^{\operatorname{mix}}=\frac{G_{F}^{2} m_{W}^{2}}{16 \pi^{2}} Q_{1}^{\mathrm{mix}}\left|V_{t b} V_{t s}^{*}\right|^{2} C_{1, t t}^{\mathrm{mix}}
$$

with the effective operator

$$
Q_{1}^{\operatorname{mix}}=\left(\bar{s}_{L} \gamma_{\mu} b_{L}\right)\left(\bar{s}_{L} \gamma^{\mu} b_{L}\right)
$$




\section{B SMEFT coefficients in the mass basis}

In the up-mass basis we absorb the unitary rotations $S_{L, R}^{u}$ between the flavor and mass basis into the Wilson coefficients. The ones of the operators (2.2) are then given as

$$
\begin{aligned}
\hat{C}_{\varphi q}^{(1) i j} & =\hat{C}_{\varphi q}^{(1) k l}\left(S_{L}^{u \dagger}\right)_{i k}\left(S_{L}^{u}\right)_{l j}, & \hat{C}_{\varphi q}^{(3) i j} & =\hat{C}_{\varphi q}^{(3) k l}\left(S_{L}^{u \dagger}\right)_{i k}\left(S_{L}^{u}\right)_{l j} \\
\hat{C}_{u B}^{i j} & =\hat{C}_{u B}^{k l}\left(S_{L}^{u \dagger}\right)_{i k}\left(S_{R}^{u}\right)_{l j}, & \hat{C}_{u W}^{i j} & =\hat{C}_{u W}^{k l}\left(S_{L}^{u \dagger}\right)_{i k}\left(S_{R}^{u}\right)_{l j}, \\
\hat{C}_{u G}^{i j} & =\hat{C}_{u G}^{k l}\left(S_{L}^{u \dagger}\right)_{i k}\left(S_{R}^{u}\right)_{l j}, & \hat{C}_{\varphi u}^{i j} & =\hat{C}_{\varphi u}^{k l}\left(S_{R}^{u \dagger}\right)_{i k}\left(S_{R}^{u}\right)_{l j},
\end{aligned}
$$

Similarly, we obtain for the coefficients of the four-fermion operators (2.3)

$$
\begin{array}{rlrl}
\hat{C}_{l q}^{(1) i j} & =C_{l q}^{(1) k l}\left(S_{L}^{u \dagger}\right)_{i k}\left(S_{L}^{u}\right)_{l j}, & \hat{C}_{l q}^{(3) i j}=C_{l q}^{(3) k l}\left(S_{L}^{u \dagger}\right)_{i k}\left(S_{L}^{u}\right)_{l j}, \\
\hat{C}_{q e}^{i j} & =C_{q e}^{k l}\left(S_{L}^{u \dagger}\right)_{i k}\left(S_{L}^{u}\right)_{l j}, & & \hat{C}_{e u}^{i j}=C_{e u}^{k l}\left(S_{R}^{u \dagger}\right)_{i k}\left(S_{R}^{u}\right)_{l j}, \\
\hat{C}_{l u}^{i j} & =C_{l u}^{k l}\left(S_{R}^{u \dagger}\right)_{i k}\left(S_{R}^{u}\right)_{l j} . & &
\end{array}
$$

\section{SMEFT operators in the mass basis}

In the up-mass eigenbasis, with coefficients defined according to eq. (B.1) we find for the effective operators in eq. (2.2)

$$
\begin{aligned}
\hat{C}_{\varphi q}^{(1) i j} \hat{O}_{\varphi q}^{(1) i j} & =\hat{C}_{\varphi q}^{(1) i j}\left(\varphi^{\dagger} i \overleftrightarrow{D}_{\mu} \varphi\right)\left(\bar{u}_{L}^{i} \gamma^{\mu} u_{L}^{\prime j}+V_{k i}^{\dagger} V_{i l} \bar{d}_{L}^{\prime k} \gamma^{\mu} d_{L}^{\prime l}\right), \\
\hat{C}_{\varphi q}^{(3) i j} \hat{O}_{\varphi q}^{(3) i j} & =\hat{C}_{\varphi q}^{(3) i j}\left(\varphi^{\dagger} i \overleftrightarrow{D}_{\mu}^{3} \varphi\right)\left(\bar{u}_{L}^{\prime i} \gamma^{\mu} u_{L}^{\prime j}-V_{k i}^{\dagger} V_{j l} \bar{d}_{L}^{k} \gamma^{\mu} d_{L}^{\prime l}\right)+\ldots \\
\hat{C}_{u B}^{i j} \hat{O}_{u B}^{i j} & =\hat{C}_{u B}^{i j}\left(\bar{u}_{L}^{\prime i} \sigma^{\mu \nu} u_{R}^{\prime j}\right) \frac{h+v}{\sqrt{2}} B_{\mu \nu}+\text { h.c. }, \\
\hat{C}_{u W}^{i j} \hat{O}_{u W}^{i j} & =\hat{C}_{u W}^{i j}\left[\left(\bar{u}_{L}^{\prime i} \sigma^{\mu \nu} u_{R}^{\prime j}\right) \frac{h+v}{\sqrt{2}} W_{\mu \nu}^{3}+V_{k i}^{\dagger}\left(\bar{d}_{L}^{\prime k} \sigma^{\mu \nu} u_{R}^{\prime j}\right) \frac{h+v}{\sqrt{2}} W_{\mu \nu}^{-}\right]+\text {h.c. }, \\
\hat{C}_{u G}^{i j} \hat{O}_{u G}^{i j} & =\hat{C}_{u G}^{i j}\left(\bar{u}_{L}^{\prime i} \sigma^{\mu \nu} T^{A} u_{R}^{\prime j}\right) \frac{h+v}{\sqrt{2}} G_{\mu \nu}^{A}+\text { h.c. }, \\
\hat{C}_{\varphi u}^{i j} \hat{O}_{\varphi u}^{i j} & =\hat{C}_{\varphi u}^{i j}\left(\varphi^{\dagger} i \overleftrightarrow{D}_{\mu} \varphi\right)\left(\bar{u}_{R}^{i} \gamma^{\mu} u_{R}^{j}\right) .
\end{aligned}
$$

Similarly, we find for the four-fermion operators in eq. (2.3) with coefficients defined in eq. (B.2)

$$
\begin{aligned}
\hat{C}_{l q}^{(1) i j} \hat{O}_{l q}^{(1) i j} & =\hat{C}_{l q}^{(1) i j}\left(\bar{l}_{L} \gamma_{\mu} l_{L}\right)\left(\bar{u}_{L}^{i} \gamma^{\mu} u_{L}^{\prime j}+V_{k i}^{\dagger} V_{i l} \bar{d}_{L}^{\prime k} \gamma^{\mu} d_{L}^{l}\right), \\
\hat{C}_{l q}^{(3) i j} \hat{O}_{l q}^{(3) i j} & =\hat{C}_{l q}^{(3) i j}\left(\bar{l}_{L} \gamma_{\mu} \tau^{3} l_{L}\right)\left(\bar{u}_{L}^{\prime i} \gamma^{\mu} u_{L}^{\prime j}-V_{k i}^{\dagger} V_{i l} \bar{d}_{L}^{\prime k} \gamma^{\mu} d_{L}^{\prime l}\right)+\ldots \\
\hat{C}_{q e}^{(1) i j} \hat{O}_{q e}^{(1) i j} & =\hat{C}_{q e}^{(1) i j}\left(\bar{e}_{R} \gamma_{\mu} e_{R}\right)\left(\bar{u}_{L}^{\prime i} \gamma^{\mu} u_{L}^{\prime j}+V_{k i}^{\dagger} V_{i l} \bar{d}_{L}^{\prime k} \gamma^{\mu} d_{L}^{\prime l}\right), \\
\hat{C}_{e u}^{i j} \hat{O}_{e u}^{i j} & =\hat{C}_{e u}^{i j}\left(\bar{e}_{R} \gamma_{\mu} e_{R}\right)\left(\bar{u}_{R}^{i i} \gamma^{\mu} u_{R}^{\prime j}\right), \\
\hat{C}_{l u}^{i j} \hat{O}_{l u}^{i j} & =\hat{C}_{l u}^{i j}\left(\bar{l}_{L} \gamma_{\mu} l_{L}\right)\left(\bar{u}_{R}^{i} \gamma^{\mu} u_{R}^{\prime j}\right)
\end{aligned}
$$

These results are in agreement with ref. [51]. 


\section{Analytic formulas for one-loop matching}

The contributions from $\tilde{C}_{\varphi q}^{(i)}$ to $C_{7}$ and $C_{8}$ are taken from [62]:

$$
\begin{aligned}
E_{7}^{\varphi q}\left(x_{t}\right) & =\frac{1}{27}\left(2 \cos ^{2} \theta_{w}+1\right), \\
E_{7}^{\varphi q(3)}\left(x_{t}\right) & =\frac{8 x_{t}^{3}+5 x_{t}^{2}-7 x_{t}}{12\left(x_{t}-1\right)^{3}}+\frac{x_{t}^{2}\left(2-3 x_{t}\right)}{2\left(1-x_{t}\right)^{4}} \log x_{t}+\frac{1}{54}\left(-4 \cos ^{2} \theta_{w}+67\right), \\
E_{8}^{\varphi q}\left(x_{t}\right) & =\frac{1}{9}\left(2 \cos ^{2} \theta_{w}+1\right), \\
E_{8}^{\varphi q(3)}\left(x_{t}\right) & =-\frac{x_{t}\left(x_{t}^{2}-5 x_{t}-2\right)}{4\left(x_{t}-1\right)^{3}}-\frac{3}{2} \frac{x_{t}^{2}}{\left(x_{t}-1\right)^{4}} \log x_{t}+\frac{1}{9}\left(2 \cos ^{2} \theta_{w}+1\right) .
\end{aligned}
$$

The remaining functions relevant for contributions from dipole operators read [51]

$$
\begin{aligned}
E_{7}^{u W}\left(x_{t}\right)= & \frac{-9 x_{t}^{3}+63 x_{t}^{2}-61 x_{t}+19}{48\left(x_{t}-1\right)^{3}}+\frac{\left(3 x_{t}^{4}-12 x_{t}^{3}-9 x_{t}^{2}+20 x_{t}-8\right) \ln \left(x_{t}\right)}{24\left(x_{t}-1\right)^{4}} \\
& +\frac{1}{8} \ln \left(\frac{m_{W}^{2}}{\mu_{W}^{2}}\right) \\
F_{7}^{u W}\left(x_{t}\right)= & \frac{x_{t}\left(2-3 x_{t}\right) \ln \left(x_{t}\right)}{4\left(x_{t}-1\right)^{4}}-\frac{3 x_{t}^{3}-17 x_{t}^{2}+4 x_{t}+4}{24\left(x_{t}-1\right)^{3}}, \\
E_{7}^{u B}\left(x_{t}\right)= & -\frac{1}{8} \ln \left(\frac{m_{W}^{2}}{\mu_{W}^{2}}\right)-\frac{\left(x_{t}+1\right)^{2}}{16\left(x_{t}-1\right)^{2}}-\frac{x_{t}^{2}\left(x_{t}-3\right) \ln \left(x_{t}\right)}{8\left(x_{t}-1\right)^{3}}, \\
F_{7}^{u B}\left(x_{t}\right)= & -\frac{1}{8}, \\
E_{8}^{u W}\left(x_{t}\right)= & \frac{3 x_{t}^{2}-13 x_{t}+4}{8\left(x_{t}-1\right)^{3}}+\frac{\left(5 x_{t}-2\right) \ln \left(x_{t}\right)}{4\left(x_{t}-1\right)^{4}} \\
F_{8}^{u W}\left(x_{t}\right)= & \frac{x_{t}^{2}-5 x_{t}-2}{8\left(x_{t}-1\right)^{3}}+\frac{3 x_{t} \ln \left(x_{t}\right)}{4\left(x_{t}-1\right)^{4}}, \\
E_{8}^{u G}\left(x_{t}\right)= & E_{7}^{u B}\left(x_{t}\right), \\
F_{8}^{u G}\left(x_{t}\right)= & F_{7}^{u B}\left(x_{t}\right) .
\end{aligned}
$$

The following functions relevant for the matching of up-type dipole operators on $C_{9}$ and $C_{10}$ are taken from ref. [51] and read

$$
\begin{aligned}
Y_{u W}\left(x_{t}\right) & =\frac{3 x_{t}}{4\left(x_{t}-1\right)}-\frac{3 x_{t}}{4\left(x_{t}-1\right)^{2}} \ln \left(x_{t}\right), \\
Z_{u W}\left(x_{t}\right) & =\frac{99 x_{t}^{3}-136 x_{t}^{2}-25 x_{t}+50}{36\left(x_{t}-1\right)^{3}}-\frac{24 x_{t}^{3}-45 x_{t}^{2}+17 x_{t}+2}{6\left(x_{t}-1\right)^{4}} \ln \left(x_{t}\right), \\
Z_{u B}\left(x_{t}\right) & =-\frac{x_{t}^{2}+3 x_{t}-2}{4\left(x_{t}-1\right)^{2}}+\frac{3 x_{t}-2}{2\left(x_{t}-1\right)^{3}} \ln \left(x_{t}\right) .
\end{aligned}
$$


Contributions for both four-fermion operators and operators with two Higgs bosons can be parametrized in terms of functions [63]

$$
\begin{aligned}
K_{0}(x, \mu) & =-\frac{x}{32}\left[\ln \frac{\mu^{2}}{m_{W}^{2}}+\frac{3(x+1)}{2(x-1)}-\frac{x^{2}-2 x+4}{(x-1)^{2}} \ln x\right], \\
K_{1}(x, \mu) & =\frac{x}{16}\left[\ln \frac{\mu^{2}}{m_{W}^{2}}+\frac{x-7}{2(x-1)}-\frac{x^{2}-2 x-2}{(x-1)^{2}} \ln x\right], \\
K_{2}(x, \mu) & =-\frac{x}{8}\left[\ln \frac{\mu^{2}}{m_{W}^{2}}+1-\ln x\right], \\
J_{2}(x) & =\frac{x}{8}, \\
J_{3}(x, \mu) & =-\frac{3}{16} x_{t}\left[\ln \frac{\mu^{2}}{m_{W}^{2}}+\frac{x_{t}+3}{2\left(x_{t}-1\right)}-\frac{x_{t}^{2}+1}{\left(x_{t}-1\right)^{2}} \ln x_{t}\right], \\
B(x) & =\frac{3}{16} x\left[\frac{1}{x-1}-\frac{1}{(x-1)^{2}} \ln x\right], \\
D(x) & =-\frac{2}{9} \ln x-\frac{x}{72}\left[\frac{82 x^{2}-151 x+63}{(x-1)^{3}}-\frac{10 x^{3}+59 x^{2}-138 x+63}{(x-1)^{4}} \ln x\right] .
\end{aligned}
$$

With these definitions, the functions appearing in the matching to $C_{9}$ and $C_{10}$ read [63]

$$
\begin{aligned}
I_{1}\left(x_{t}\right)= & -J_{2}\left(x_{t}\right)-2 K_{0}\left(x_{t}, \mu_{W}\right) \\
= & \frac{x_{t}}{16}\left[\ln \frac{\mu^{2}}{m_{W}^{2}}-\frac{x_{t}-7}{2\left(x_{t}-1\right)}-\frac{x_{t}^{2}-2 x_{t}+4}{\left(x_{t}-1\right)^{2}} \ln x_{t}\right] . \\
I_{2}\left(x_{t}\right)= & J_{2}\left(x_{t}\right)+K_{2}\left(x_{t}, \mu_{w}\right)+K_{0}\left(x_{t}, \mu\right) \\
= & -\frac{x_{t}}{32}\left(5 \ln \frac{\mu_{W}^{2}}{m_{W}^{2}}+\frac{3\left(x_{t}+1\right)}{2\left(x_{t}-1\right)}-\frac{5 x_{t}^{2}-10 x_{t}+8}{\left(x_{t}-1\right)^{2}} \ln x_{t}\right), \\
I^{l q}\left(x_{t}\right)= & -J_{2}\left(x_{t}\right)+K_{2}\left(x_{t}, \mu_{w}\right)+K_{0}\left(x_{t}, \mu\right) \\
= & -\frac{x_{t}}{32}\left(8+5 \ln \frac{\mu_{W}^{2}}{m_{W}^{2}}+\frac{3\left(x_{t}+1\right)}{2\left(x_{t}-1\right)}-\frac{5 x_{t}^{2}-10 x_{t}+8}{\left(x_{t}-1\right)^{2}} \ln x_{t}\right), \\
I_{1}^{\varphi q}\left(x_{t}\right)= & \left(-1+4 \sin ^{2} \theta_{w}\right)\left(J_{2}\left(x_{t}\right)+2 J_{3}\left(x_{t}\right)-K_{1}\left(x_{t}\right)-3 K_{0}\left(x_{t}, \mu\right)\right) \\
& +2\left(B\left(x_{t}\right)+2 \sin ^{2} \theta_{w} D\left(x_{t}\right)\right) \\
= & \left(-1+4 \sin ^{2} \theta_{w}\right) \frac{x_{t}}{32}\left(4-11 \ln \frac{\mu_{W}^{2}}{m_{W}^{2}}-\frac{5 x_{t}+13}{2\left(x_{t}-1\right)}+\frac{11 x_{t}^{2}+2 x_{t}-4}{\left(x_{t}-1\right)^{2}} \ln x_{t}\right) \\
& +\frac{3}{8} x\left[\frac{1}{x_{t}-1}-\frac{1}{\left(x_{t}-1\right)^{2}} \ln x_{t}\right]+\sin ^{2} \theta_{w}\left\{-\frac{8}{9} \ln x_{t}\right. \\
& \left.-\frac{x_{t}}{18}\left[\frac{82 x_{t}^{2}-151 x_{t}+63}{\left(x_{t}-1\right)^{3}}-\frac{10 x_{t}^{3}+59 x_{t}^{2}-138 x_{t}+63}{\left(x_{t}-1\right)^{4}} \ln x_{t}\right]\right\},
\end{aligned}
$$




$$
\begin{aligned}
I_{2}^{\varphi q}\left(x_{t}\right) & =\left(J_{2}\left(x_{t}\right)+2 J_{3}\left(x_{t}\right)-K_{1}\left(x_{t}\right)-3 K_{0}\left(x_{t}, \mu\right)\right)+2 B\left(x_{t}\right) \\
& =\frac{x_{t}}{32}\left(4-11 \ln \frac{\mu_{W}^{2}}{m_{W}^{2}}-\frac{5 x_{t}+37}{2\left(x_{t}-1\right)}+\frac{11 x_{t}^{2}+2 x_{t}+8}{\left(x_{t}-1\right)^{2}} \ln x_{t}\right),
\end{aligned}
$$

where we neglected CKM-suppressed contributions $\sim\left|V_{t s}\right|^{2}, \sim\left|V_{t d}\right|^{2}$, which are smaller by a factor of at least $\sim 10^{-3}$.

The functions $H_{i}$ relevant for the matching of $\tilde{C}_{\varphi q}^{(1)}$ and $\tilde{C}_{\varphi q}^{(3)}$ onto $C_{1}^{\text {mix }}$ read [61]

$$
\begin{aligned}
& H_{1}\left(x_{t}\right)=-\frac{x_{t}-7}{4\left(x_{t}-1\right)}-\frac{x_{t}^{2}-2 x_{t}+4}{2\left(x_{t}-1\right)^{2}} \ln x_{t}, \\
& H_{1}\left(x_{t}\right)=+\frac{7 x_{t}-25}{4\left(x_{t}-1\right)}-\frac{x_{t}^{2}-14 x_{t}+4}{2\left(x_{t}-1\right)^{2}} \ln x_{t} .
\end{aligned}
$$

Finally, functions relevant for the matching of SMEFT coefficients onto $C_{L}$ at one-loop level are taken from [62]. Here, we give results with all evanescent coefficients set to 1 :

$$
\begin{aligned}
& I_{u W}^{\nu}=\frac{m_{t}}{m_{W} \sin ^{2} \theta_{w}}\left(-\frac{3\left(x_{t}-2\right)}{4 \sqrt{2}\left(x_{t}-1\right)}-\frac{3 x_{t} \ln x_{t}}{\sqrt{2}\left(x_{t}-1\right)^{2}}\right), \\
& I_{\varphi q}^{\nu(1)}=\frac{1}{\sin ^{2} \theta_{w}}\left(\frac{x_{t}}{8}-\frac{3 x_{t}\left(x_{t}+1\right)}{32\left(x_{t}-1\right)}-\frac{x_{t}\left(x_{t}^{2}-2 x_{t}+4\right) \ln \frac{\mu_{W}^{2}}{m_{t}^{2}}}{16\left(x_{t}-1\right)^{2}}+\frac{3 x_{t} \ln \frac{\mu_{W}^{2}}{m_{W}^{2}}}{16\left(x_{t}-1\right)^{2}}\right. \\
& \left.+\frac{\left(2 m_{W}^{2}+m_{Z}^{2}\right)}{8 m_{W}^{2}}-\frac{3 x_{t} \ln \frac{\mu_{W}^{2}}{m_{t}^{2}}}{8}\right) \\
& I_{\varphi q}^{\nu(3)}=\frac{1}{\sin ^{2} \theta_{w}}\left(-\frac{x_{t}}{8}+\frac{5 x_{t}\left(x_{t}-7\right)}{32\left(x_{t}-1\right)}+\frac{x_{t}\left(7 x_{t}^{2}-2 x_{t}-20\right) \ln \frac{\mu_{W}^{2}}{m_{t}^{2}}}{16\left(x_{t}-1\right)^{2}}-\frac{3 x_{t}\left(4 x_{t}-9\right) \ln \frac{\mu_{W}^{2}}{m_{W}^{2}}}{16\left(x_{t}-1\right)^{2}}\right. \\
& \left.+\frac{19 m_{W}^{2}+m_{Z}^{2}}{8 m_{W}^{2}}-\frac{3 \ln \frac{m u_{W}^{2}}{m_{W}^{2}}}{8}+\frac{3 m_{W}^{2} \ln \frac{\mu_{W}^{2}}{m_{W}^{2}}}{4 m_{W}}\right) \\
& I_{l u}^{\nu}=\frac{1}{\sin ^{2} \theta_{w}}\left(-\frac{x_{t}\left(x_{t}-7\right)}{32\left(x_{t}-1\right)}+\frac{\left(x_{t}^{3}-2 x_{t}^{2}+4 x_{t}\right) \ln \frac{\mu_{w}^{2}}{m_{t}^{2}}}{16\left(x_{t}-1\right)^{2}}-\frac{3 x_{t} \ln \frac{\mu_{w}^{2}}{m_{W}^{2}}}{16\left(x_{t}-1\right)^{2}}\right) \\
& I_{l q}^{\nu(1)}=\frac{1}{\sin ^{2} \theta_{w}}\left(\frac{x_{t}}{8}-\frac{3 x_{t}\left(x_{t}+1\right)}{32\left(x_{t}-1\right)}-\frac{x_{t}\left(x_{t}^{2}-2 x_{t}+4\right) \ln \frac{\mu_{W}^{2}}{m_{t}^{2}}}{16\left(x_{t}-1\right)^{2}}+\frac{3 x_{t} \ln \frac{\mu_{W}^{2}}{m_{W}^{2}}}{16\left(x_{t}-1\right)^{2}}\right. \\
& \left.+\frac{11\left(2 m_{W}^{2}+m_{Z}^{2}\right)}{48 m_{W}^{2}}+\frac{\left(2 m_{W}^{2}+m_{Z}^{2}\right) \ln \frac{\mu_{W}^{2}}{m_{Z}^{2}}}{8 m_{W}}\right)
\end{aligned}
$$




$$
\begin{aligned}
I_{l q}^{\nu(3)}= & \frac{1}{\sin ^{2} \theta_{w}}\left(\frac{x_{t}}{8}-\frac{3 x_{t}\left(x_{t}+1\right)}{32\left(x_{t}-1\right)}-\frac{x_{t}\left(x_{t}^{2}-26 x_{t}+28\right) \ln \frac{\mu_{W}^{2}}{m_{t}^{2}}}{16\left(x_{t}-1\right)^{2}}+\frac{3 x_{t}\left(8 x_{t}-9\right) \ln \frac{\mu_{W}^{2}}{m_{W}^{2}}}{16\left(x_{t}-1\right)^{2}}\right. \\
& \left.+\frac{-154 m_{W}^{2}-11 m_{Z}^{2}}{48 m_{W}^{2}}-\frac{3 \ln \frac{m u_{W}^{2}}{m_{W}^{2}}}{2}-\frac{\left(2 m_{W}^{2}+m_{Z}^{2}\right) \ln \frac{\mu_{W}^{2}}{m_{Z}^{2}}}{8 m_{W}}\right) .
\end{aligned}
$$

\section{E Numerical matching conditions}

The numerical values of the tree-level matching conditions in eq. (2.12) read at $\mu_{W}=m_{W}$

$$
\begin{aligned}
\Delta C_{9}^{\text {tree }} & =402.1\left[\tilde{C}_{l q}^{1}+\tilde{C}_{l q}^{3}+\tilde{C}_{q e}\right]-44.53\left(\tilde{C}_{\varphi q}^{1}+\tilde{C}_{\varphi q}^{3}\right), \\
\Delta C_{10}^{\text {tree }} & =402.1\left[-\tilde{C}_{l q}^{1}-\tilde{C}_{l q}^{3}+\tilde{C}_{q e}+\tilde{C}_{\varphi q}^{1}+\tilde{C}_{\varphi q}^{3}\right], \\
\Delta C_{L}^{\text {tree }} & =402.1\left[\tilde{C}_{l q}^{1}-\tilde{C}_{l q}^{3}+\tilde{C}_{\varphi q}^{1}+\tilde{C}_{\varphi q}^{3}\right] .
\end{aligned}
$$

For the one-loop contributions in eqs. (2.13)-(2.18) we obtain at $\mu_{W}=m_{W}$

$$
\begin{aligned}
\Delta C_{7}^{\text {loop }}= & -2.31 \tilde{C}_{u B}+0.0925 \tilde{C}_{u W}--0.132 \tilde{C}_{\varphi q}^{(1)}+1.12 \tilde{C}_{\varphi q}^{(3)}, \\
\Delta C_{8}^{\text {loop }}= & -0.669 \tilde{C}_{u G}+0.271 \tilde{C}_{u W}+0.392 \tilde{C}_{\varphi q}^{(1)}+1.05 \tilde{C}_{\varphi q}^{(3)}, \\
\Delta C_{9}^{\text {loop }}= & 2.170 \tilde{C}_{u W}+2.512 \tilde{C}_{u B}-1.81 \tilde{C}_{\varphi q}^{(1)}-1.96 \tilde{C}_{\varphi q}^{(3)}+0.148 \tilde{C}_{\varphi u} \\
& -1.898\left(\tilde{C}_{e u}+\tilde{C}_{l u}\right)-2.242\left(\tilde{C}_{l q}^{(1)}-\tilde{C}_{q e}\right)-4.444 \tilde{C}_{l q}^{(3)}, \\
\Delta C_{10}^{\text {loop }}= & -7.54 \tilde{C}_{u W}+12.8 \tilde{C}_{\varphi q}^{(1)}-3.43 \tilde{C}_{\varphi q}^{(3)}-1.90 \tilde{C}_{\varphi u}-1.90\left(\tilde{C}_{e u}-\tilde{C}_{l u}\right) \\
& -2.242\left(\tilde{C}_{l q}^{(1)}-\tilde{C}_{q e}\right)+4.444 \tilde{C}_{l q}^{(3)}, \\
\Delta C_{L}^{\text {loop }}= & -2.88 \tilde{C}_{u W}+14.9 \tilde{C}_{\varphi q}^{(1)}+0.332 \tilde{C}_{\varphi q}^{(3)}-1.90\left(\tilde{C}_{\varphi u}+\tilde{C}_{l u}\right) \\
& +4.622 \tilde{C}_{l q}^{(1)}+1.033 \tilde{C}_{l q}^{(3)}, \\
\Delta C_{1, t t}^{\text {mix, loop }}= & 4.12 \tilde{C}_{u W}+14.8 \tilde{C}_{\varphi q}^{(1)}+11.6 \tilde{C}_{\varphi q}^{(3)} .
\end{aligned}
$$




\section{F Auxiliary plots}
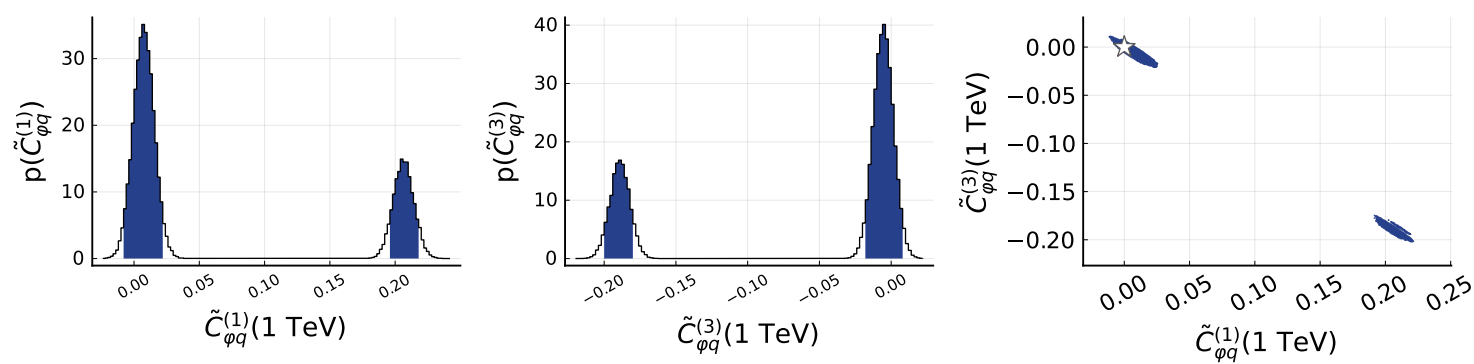

Figure 13. One-dimensional (left, middle) and two-dimensional (right) projections of the posterior distribution for $\tilde{C}_{\varphi q}^{(1)}$ and $\tilde{C}_{\varphi q}^{(3)}$. Results are obtained for a fit of eight SMEFT coefficients in eq. (4.3) to the combined set of present top-quark, $Z b \bar{b}$, and $B$ physics data. The star in the rightmost plot denotes the SM point.
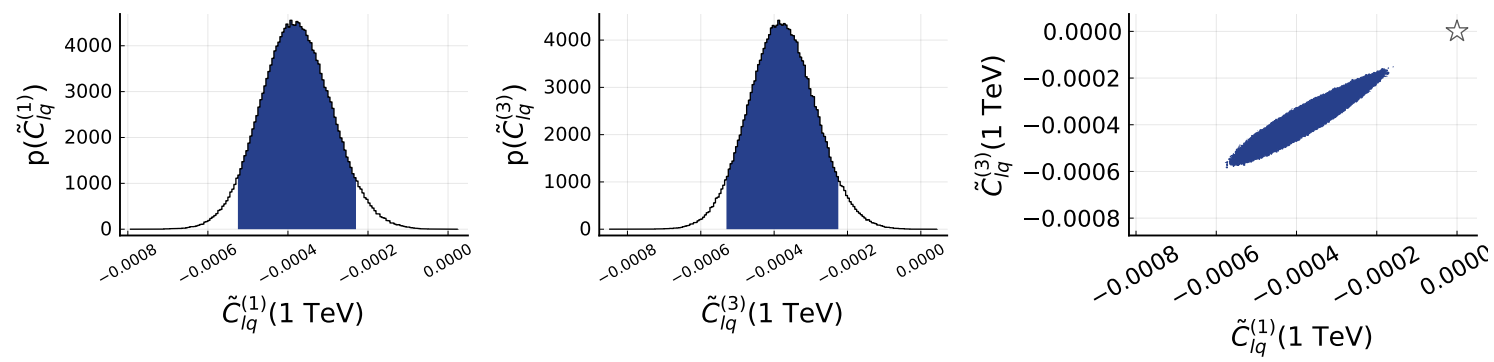

Figure 14. One-dimensional (left, middle) and two-dimensional (right) projections of the posterior distribution for $\tilde{C}_{l q}^{(1)}$ and $\tilde{C}_{l q}^{(3)}$. Results are obtained for a fit of all eleven coefficients in eq. (2.8) to the combined set of present data, near future projections, and CLIC projections for top-quark observables. The star in the rightmost plot denotes the SM point.
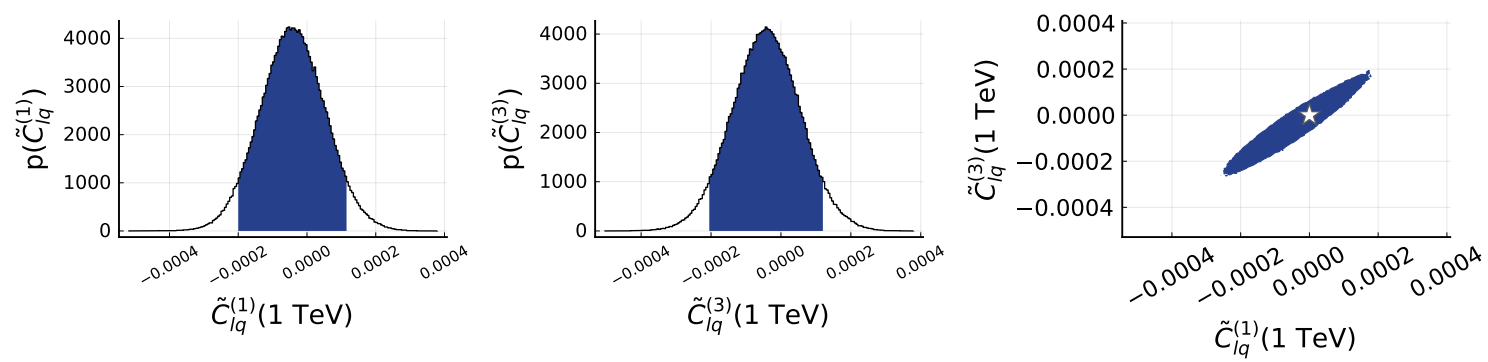

Figure 15. The same as in figure 14 but assuming SM central values for $b \rightarrow s$ observables at Belle II.

Open Access. This article is distributed under the terms of the Creative Commons Attribution License (CC-BY 4.0), which permits any use, distribution and reproduction in any medium, provided the original author(s) and source are credited. 


\section{References}

[1] C. Degrande, F. Maltoni, K. Mimasu, E. Vryonidou and C. Zhang, Single-top associated production with a $Z$ or $H$ boson at the LHC: the SMEFT interpretation, JHEP 10 (2018) 005 [arXiv: 1804.07773] [INSPIRE].

[2] M. Chala, J. Santiago and M. Spannowsky, Constraining four-fermion operators using rare top decays, JHEP 04 (2019) 014 [arXiv: 1809.09624] [INSPIRE].

[3] G. Durieux, F. Maltoni and C. Zhang, Global approach to top-quark flavor-changing interactions, Phys. Rev. D 91 (2015) 074017 [arXiv:1412.7166] [INSPIRE].

[4] J.A. Aguilar-Saavedra, Effective four-fermion operators in top physics: A Roadmap, Nucl. Phys. B 843 (2011) 638 [Erratum ibid. 851 (2011) 443] [arXiv:1008.3562] [INSPIRE].

[5] J. D'Hondt, A. Mariotti, K. Mimasu, S. Moortgat and C. Zhang, Learning to pinpoint effective operators at the LHC: a study of the $t \bar{t} b \bar{b}$ signature, JHEP 11 (2018) 131 [arXiv: 1807.02130] [INSPIRE].

[6] G. Durieux, J. Gu, E. Vryonidou and C. Zhang, Probing top-quark couplings indirectly at Higgs factories, Chin. Phys. C 42 (2018) 123107 [arXiv: 1809.03520] [InSPIRE].

[7] A. Buckley et al., Global fit of top quark effective theory to data, Phys. Rev. D 92 (2015) 091501 [arXiv: 1506.08845] [INSPIRE].

[8] A. Buckley et al., Constraining top quark effective theory in the LHC Run II era, JHEP 04 (2016) 015 [arXiv:1512.03360] [INSPIRE].

[9] M. de Beurs, E. Laenen, M. Vreeswijk and E. Vryonidou, Effective operators in t-channel single top production and decay, Eur. Phys. J. C 78 (2018) 919 [arXiv:1807.03576] [INSPIRE].

[10] S. Brown et al., TopFitter: Fitting top-quark Wilson Coefficients to Run II data, PoS ICHEP2018 (2019) 293 [arXiv:1901.03164] [INSPIRE].

[11] D. Barducci et al., Interpreting top-quark LHC measurements in the standard-model effective field theory, arXiv: 1802.07237 [INSPIRE].

[12] N.P. Hartland et al., A Monte Carlo global analysis of the Standard Model Effective Field Theory: the top quark sector, JHEP 04 (2019) 100 [arXiv:1901.05965] [INSPIRE].

[13] F. Maltoni, L. Mantani and K. Mimasu, Top-quark electroweak interactions at high energy, JHEP 10 (2019) 004 [arXiv: 1904.05637] [INSPIRE].

[14] G. Durieux et al., The electro-weak couplings of the top and bottom quarks - Global fit and future prospects, JHEP 12 (2019) 098 [Erratum JHEP 01 (2021) 195] [arXiv:1907.10619] [INSPIRE].

[15] T. Neumann and Z.E. Sullivan, Off-Shell Single-Top-Quark Production in the Standard Model Effective Field Theory, JHEP 06 (2019) 022 [arXiv:1903.11023] [INSPIRE].

[16] I. Brivio et al., O new physics, where art thou? A global search in the top sector, JHEP 02 (2020) 131 [arXiv:1910.03606] [INSPIRE].

[17] J.A. Dror, M. Farina, E. Salvioni and J. Serra, Strong tW Scattering at the LHC, JHEP 01 (2016) 071 [arXiv: 1511.03674] [INSPIRE].

[18] S. Bißmann, J. Erdmann, C. Grunwald, G. Hiller and K. Kröninger, Constraining top-quark couplings combining top-quark and B decay observables, Eur. Phys. J. C 80 (2020) 136 [arXiv: 1909.13632] [INSPIRE]. 
[19] R. Aoude, T. Hurth, S. Renner and W. Shepherd, The impact of flavour data on global fits of the MFV SMEFT, JHEP 12 (2020) 113 [arXiv:2003.05432] [INSPIRE].

[20] P.J. Fox, Z. Ligeti, M. Papucci, G. Perez and M.D. Schwartz, Deciphering top flavor violation at the LHC with B factories, Phys. Rev. D 78 (2008) 054008 [arXiv:0704.1482] [INSPIRE].

[21] B. Grzadkowski and M. Misiak, Anomalous Wtb coupling effects in the weak radiative B-meson decay, Phys. Rev. D 78 (2008) 077501 [Erratum ibid. 84 (2011) 059903] [arXiv:0802.1413] [INSPIRE].

[22] J. Drobnak, S. Fajfer and J.F. Kamenik, Probing anomalous tWb interactions with rare $B$ decays, Nucl. Phys. B 855 (2012) 82 [arXiv:1109.2357] [InSPIRE].

[23] J. Brod, A. Greljo, E. Stamou and P. Uttayarat, Probing anomalous $t \bar{t} Z$ interactions with rare meson decays, JHEP 02 (2015) 141 [arXiv: 1408.0792] [INSPIRE].

[24] S. Bifani, S. Descotes-Genon, A. Romero Vidal and M.-H. Schune, Review of Lepton Universality tests in B decays, J. Phys. G 46 (2019) 023001 [arXiv: 1809.06229] [InSPIRE].

[25] M.S. Amjad et al., A precise characterisation of the top quark electro-weak vertices at the ILC, Eur. Phys. J. C 75 (2015) 512 [arXiv: 1505.06020] [INSPIRE].

[26] M.S. Amjad et al., A precise determination of top quark electro-weak couplings at the ILC operating at $\sqrt{s}=500 \mathrm{GeV}$, arXiv: 1307.8102 [INSPIRE].

[27] CLICDP collaboration, Top-Quark Physics at the CLIC Electron-Positron Linear Collider, JHEP 11 (2019) 003 [arXiv:1807.02441] [INSPIRE].

[28] FCC collaboration, FCC-ee: The Lepton Collider: Future Circular Collider Conceptual Design Report. Volume 2, Eur. Phys. J. Spec. Top. 228 (2019) 261.

[29] G.L. Kane, G.A. Ladinsky and C.P. Yuan, Using the Top Quark for Testing Standard Model Polarization and CP Predictions, Phys. Rev. D 45 (1992) 124 [InSPIRE].

[30] D. Atwood and A. Soni, Analysis for magnetic moment and electric dipole moment form-factors of the top quark via $e^{+} e^{-} \rightarrow t \bar{t}$, Phys. Rev. D 45 (1992) 2405 [inSPIRE].

[31] B. Grzadkowski, Z. Hioki and M. Szafranski, Four-Fermi effective operators in top-quark production and decay, Phys. Rev. D 58 (1998) 035002 [hep-ph/9712357] [INSPIRE].

[32] L. Brzezinski, B. Grzadkowski and Z. Hioki, Effects of nonstandard interactions for the energy spectrum of secondary leptons in $e^{+} e^{-} \rightarrow t \bar{t}$, Int. J. Mod. Phys. A 14 (1999) 1261 [hep-ph/9710358] [INSPIRE].

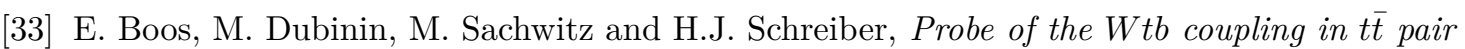
production at linear colliders, Eur. Phys. J. C 16 (2000) 269 [hep-ph/0001048] [INSPIRE].

[34] M. Jezabek, T. Nagano and Y. Sumino, Probe of CP-violation in $e^{+} e^{-} \rightarrow t \bar{t}$ near threshold, Phys. Rev. D 62 (2000) 014034 [hep-ph/0001322] [INSPIRE].

[35] B. Grzadkowski and Z. Hioki, Optimal observable analysis of the angular and energy distributions for top quark decay products at polarized linear colliders, Nucl. Phys. B $\mathbf{5 8 5}$ (2000) 3 [Corrigendum ibid. 894 (2015) 585] [hep-ph/0004223] [INSPIRE].

[36] P. Janot, Top-quark electroweak couplings at the FCC-ee, JHEP 04 (2015) 182 [arXiv: 1503.01325] [INSPIRE].

[37] R. Röntsch and M. Schulze, Probing top-Z dipole moments at the LHC and ILC, JHEP 08 (2015) 044 [arXiv: 1501.05939] [InSPIRE]. 
[38] P.H. Khiem, E. Kou, Y. Kurihara and F. Le Diberder, Probing New Physics using top quark polarization in the $e^{+} e^{-} \rightarrow t \bar{t}$ process at future Linear Colliders, arXiv:1503.04247 [INSPIRE].

[39] C. Englert and M. Russell, Top quark electroweak couplings at future lepton colliders, Eur. Phys. J. C 77 (2017) 535 [arXiv:1704.01782] [INSPIRE].

[40] G. Durieux, M. Perelló, M. Vos and C. Zhang, Global and optimal probes for the top-quark effective field theory at future lepton colliders, JHEP 10 (2018) 168 [arXiv:1807.02121] [INSPIRE].

[41] Q.-H. Cao and B. Yan, Determining $V_{t b}$ at electron-positron colliders, Phys. Rev. D 92 (2015) 094018 [arXiv: 1507.06204] [INSPIRE].

[42] ATLAS and CMS collaborations, Addendum to the report on the physics at the $H L-L H C$, and perspectives for the HE-LHC: Collection of notes from ATLAS and CMS, in CERN Yellow Reports: Monographs 7, CERN, Geneva Switzerland (2019) [arXiv:1902.10229] [INSPIRE].

[43] Belle-II collaboration, The Belle II Physics Book, Prog. Theor. Exp. Phys. 2019 (2019) 123C01 [Erratum ibid. 2020 (2020) 029201] [arXiv: 1808.10567] [INSPIRE].

[44] S. Weinberg, Phenomenological Lagrangians, Physica A 96 (1979) 327 [INSPIRE].

[45] W. Buchmüller and D. Wyler, Effective Lagrangian Analysis of New Interactions and Flavor Conservation, Nucl. Phys. B 268 (1986) 621 [INSPIRE].

[46] C. Degrande et al., Effective Field Theory: A Modern Approach to Anomalous Couplings, Annals Phys. 335 (2013) 21 [arXiv:1205.4231] [INSPIRE].

[47] A. Kobach, Baryon Number, Lepton Number, and Operator Dimension in the Standard Model, Phys. Lett. B 758 (2016) 455 [arXiv: 1604.05726] [INSPIRE].

[48] B. Grzadkowski, M. Iskrzynski, M. Misiak and J. Rosiek, Dimension-Six Terms in the Standard Model Lagrangian, JHEP 10 (2010) 085 [arXiv: 1008.4884] [INSPIRE].

[49] S. Bißmann, J. Erdmann, C. Grunwald, G. Hiller and K. Kröninger, Correlating uncertainties in global analyses within SMEFT matters, Phys. Rev. D 102 (2020) 115019 [arXiv: 1912.06090] [INSPIRE].

[50] Particle Data collaboration, Review of Particle Physics, Prog. Theor. Exp. Phys. 2020 (2020) 083C01 [INSPIRE].

[51] J. Aebischer, A. Crivellin, M. Fael and C. Greub, Matching of gauge invariant dimension-six operators for $b \rightarrow s$ and $b \rightarrow c$ transitions, JHEP 05 (2016) 037 [arXiv: 1512.02830] [INSPIRE].

[52] R. Bause, H. Gisbert, M. Golz and G. Hiller, Lepton universality and lepton flavor conservation tests with dineutrino modes, arXiv:2007.05001 [INSPIRE].

[53] G. Hiller, A Challenge to Lepton Universality, Physics 7 (2014) 102 [INSPIRE].

[54] E.E. Jenkins, A.V. Manohar and M. Trott, Naive Dimensional Analysis Counting of Gauge Theory Amplitudes and Anomalous Dimensions, Phys. Lett. B 726 (2013) 697 [arXiv: 1309.0819] [INSPIRE].

[55] E.E. Jenkins, A.V. Manohar and M. Trott, Renormalization Group Evolution of the Standard Model Dimension Six Operators I: Formalism and $\lambda$ Dependence, JHEP 10 (2013) 087 [arXiv: 1308.2627] [INSPIRE]. 
[56] E.E. Jenkins, A.V. Manohar and M. Trott, Renormalization Group Evolution of the Standard Model Dimension Six Operators II: Yukawa Dependence, JHEP 01 (2014) 035 [arXiv: 1310.4838] [INSPIRE].

[57] R. Alonso, E.E. Jenkins, A.V. Manohar and M. Trott, Renormalization Group Evolution of the Standard Model Dimension Six Operators III: Gauge Coupling Dependence and Phenomenology, JHEP 04 (2014) 159 [arXiv:1312.2014] [INSPIRE].

[58] J. Aebischer, J. Kumar and D.M. Straub, Wilson: a Python package for the running and matching of Wilson coefficients above and below the electroweak scale, Eur. Phys. J. C 78 (2018) 1026 [arXiv: 1804.05033] [INSPIRE].

[59] A.J. Buras, J. Girrbach-Noe, C. Niehoff and D.M. Straub, $B \rightarrow K^{(*)} \nu \bar{\nu}$ decays in the Standard Model and beyond, JHEP 02 (2015) 184 [arXiv:1409.4557] [INSPIRE].

[60] G. Buchalla, G. Hiller and G. Isidori, Phenomenology of nonstandard $Z$ couplings in exclusive semileptonic $b \rightarrow s$ transitions, Phys. Rev. D 63 (2000) 014015 [hep-ph/0006136] [INSPIRE].

[61] C. Bobeth, A.J. Buras, A. Celis and M. Jung, Yukawa enhancement of Z-mediated new physics in $\Delta S=2$ and $\Delta B=2$ processes, JHEP 07 (2017) 124 [arXiv:1703.04753] [INSPIRE].

[62] W. Dekens and P. Stoffer, Low-energy effective field theory below the electroweak scale: matching at one loop, JHEP 10 (2019) 197 [arXiv: 1908.05295] [INSPIRE].

[63] M. Endo, S. Mishima and D. Ueda, Revisiting electroweak radiative corrections to $b \rightarrow \mathrm{sl \ell}$ in SMEFT, JHEP 05 (2021) 050 [arXiv:2012.06197] [INSPIRE].

[64] T. Hurth, S. Renner and W. Shepherd, Matching for FCNC effects in the flavour-symmetric SMEFT, JHEP 06 (2019) 029 [arXiv:1903.00500] [INSPIRE].

[65] D.M. Straub, flavio: a Python package for flavour and precision phenomenology in the Standard Model and beyond, arXiv:1810.08132 [INSPIRE].

[66] J. Alwall et al., The automated computation of tree-level and next-to-leading order differential cross sections, and their matching to parton shower simulations, JHEP 07 (2014) 079 [arXiv: 1405.0301] [InSPIRE].

[67] T. Sjöstrand et al., An introduction to PYTHIA 8.2, Comput. Phys. Commun. 191 (2015) 159 [arXiv: 1410.3012] [INSPIRE].

[68] E. Conte, B. Fuks and G. Serret, MadAnalysis 5, A User-Friendly Framework for Collider Phenomenology, Comput. Phys. Commun. 184 (2013) 222 [arXiv:1206.1599] [InSPIRE].

[69] E. Conte, B. Dumont, B. Fuks and C. Wymant, Designing and recasting LHC analyses with MadAnalysis 5, Eur. Phys. J. C $\mathbf{7 4}$ (2014) 3103 [arXiv:1405.3982] [InSPIRE].

[70] B. Dumont et al., Toward a public analysis database for LHC new physics searches using MadAnalysis 5, Eur. Phys. J. C 75 (2015) 56 [arXiv:1407.3278] [InSPIRE].

[71] M. Cacciari, G.P. Salam and G. Soyez, The anti- $k_{t}$ jet clustering algorithm, JHEP 04 (2008) 063 [arXiv: 0802.1189] [INSPIRE].

[72] M. Cacciari, G.P. Salam and G. Soyez, FastJet User Manual, Eur. Phys. J. C 72 (2012) 1896 [arXiv:1111.6097] [INSPIRE].

[73] C. Zhang and S. Willenbrock, Effective-Field-Theory Approach to Top-Quark Production and Decay, Phys. Rev. D 83 (2011) 034006 [arXiv:1008.3869] [InSPIRE]. 
[74] C. Zhang, Effective field theory approach to top-quark decay at next-to-leading order in QCD, Phys. Rev. D 90 (2014) 014008 [arXiv:1404.1264] [INSPIRE].

[75] L. Di Luzio, M. Kirk, A. Lenz and T. Rauh, $\Delta M_{s}$ theory precision confronts flavour anomalies, JHEP 12 (2019) 009 [arXiv:1909.11087] [INSPIRE].

[76] F. Feruglio, P. Paradisi and A. Pattori, On the Importance of Electroweak Corrections for B Anomalies, JHEP 09 (2017) 061 [arXiv:1705.00929] [INSPIRE].

[77] N. Castro, J. Erdmann, C. Grunwald, K. Kröninger and N.-A. Rosien, EFTfitter - A tool for interpreting measurements in the context of effective field theories, Eur. Phys. J. C 76 (2016) 432 [arXiv:1605.05585] [INSPIRE].

[78] O. Schulz et al., BAT.jl-A Julia-based tool for Bayesian inference, SN Comput. Sci. 2 (2021) 210 [arXiv:2008.03132] [INSPIRE].

[79] ATLAS and CMS collaborations, Combinations of single-top-quark production cross-section measurements and $\left|f_{L V} V_{t b}\right|$ determinations at $\sqrt{s}=7$ and 8 TeV with the ATLAS and CMS experiments, JHEP 05 (2019) 088 [arXiv:1902.07158] [INSPIRE].

[80] HFLAV collaboration, Averages of b-hadron, c-hadron, and $\tau$-lepton properties as of 2018, Eur. Phys. J. C 81 (2021) 226 [arXiv:1909.12524] [INSPIRE].

[81] ATLAS collaboration, Measurements of inclusive and differential fiducial cross-sections of $t \bar{t} \gamma$ production in leptonic final states at $\sqrt{s}=13$ TeV in ATLAS, Eur. Phys. J. C 79 (2019) 382 [arXiv:1812.01697] [INSPIRE].

[82] K. Melnikov, M. Schulze and A. Scharf, QCD corrections to top quark pair production in association with a photon at hadron colliders, Phys. Rev. D 83 (2011) 074013 [arXiv:1102.1967] [INSPIRE].

[83] CMS collaboration, Measurement of top quark pair production in association with a $Z$ boson in proton-proton collisions at $\sqrt{s}=13 \mathrm{TeV}$, JHEP 03 (2020) 056 [arXiv: 1907.11270] [INSPIRE].

[84] S. Frixione, V. Hirschi, D. Pagani, H.S. Shao and M. Zaro, Electroweak and QCD corrections to top-pair hadroproduction in association with heavy bosons, JHEP 06 (2015) 184 [arXiv: 1504.03446] [INSPIRE].

[85] LHC Higgs Cross Section Working Group, Handbook of LHC Higgs Cross Sections: 4. Deciphering the Nature of the Higgs Sector, arXiv:1610.07922 [INSPIRE].

[86] R. Frederix, S. Frixione, V. Hirschi, D. Pagani, H.S. Shao and M. Zaro, The automation of next-to-leading order electroweak calculations, JHEP 07 (2018) 185 [arXiv: 1804.10017] [INSPIRE].

[87] ATLAS collaboration, Measurement of the $t \bar{t}$ production cross-section and lepton differential distributions in e $\mu$ dilepton events from $p p$ collisions at $\sqrt{s}=13$ TeV with the ATLAS detector, Eur. Phys. J. C 80 (2020) 528 [arXiv:1910.08819] [InSPIRE].

[88] M. Czakon and A. Mitov, Top ++ : A Program for the Calculation of the Top-Pair Cross-Section at Hadron Colliders, Comput. Phys. Commun. 185 (2014) 2930 [arXiv:1112.5675] [INSPIRE].

[89] ATLAS collaboration, Measurement of the $W$ boson polarisation in $t \bar{t}$ events from $p p$ collisions at $\sqrt{s}=8 \mathrm{TeV}$ in the lepton + jets channel with ATLAS, Eur. Phys. J. C 77 (2017) 264 [Erratum ibid. 79 (2019) 19] [arXiv:1612.02577] [INSPIRE].

[90] A. Czarnecki, J.G. Korner and J.H. Piclum, Helicity fractions of $W$ bosons from top quark decays at NNLO in QCD, Phys. Rev. D 81 (2010) 111503 [arXiv:1005.2625] [InSPIRE]. 
[91] ATLAS collaboration, Direct top-quark decay width measurement in the t $\bar{t}$ lepton + jets channel at $\sqrt{s}=8 \mathrm{TeV}$ with the ATLAS experiment, Eur. Phys. J. C 78 (2018) 129 [arXiv: 1709.04207] [INSPIRE].

[92] J. Gao, C.S. Li and H.X. Zhu, Top Quark Decay at Next-to-Next-to Leading Order in QCD, Phys. Rev. Lett. 110 (2013) 042001 [arXiv:1210.2808] [INSPIRE].

[93] ALEPH et al. collaborations, Precision electroweak measurements on the $Z$ resonance, Phys. Rept. 427 (2006) 257 [hep-ex/0509008] [INSPIRE].

[94] M. Misiak et al., Updated NNLO QCD predictions for the weak radiative B-meson decays, Phys. Rev. Lett. 114 (2015) 221801 [arXiv: 1503.01789] [INSPIRE].

[95] BABAR collaboration, Measurement of the $B \rightarrow X_{s} l^{+} l^{-}$branching fraction and search for direct CP-violation from a sum of exclusive final states, Phys. Rev. Lett. 112 (2014) 211802 [arXiv: 1312.5364] [INSPIRE].

[96] T. Huber, T. Hurth and E. Lunghi, Inclusive $\bar{B} \rightarrow X_{s} \ell^{+} \ell^{-}$: complete angular analysis and a thorough study of collinear photons, JHEP 06 (2015) 176 [arXiv: 1503.04849] [INSPIRE].

[97] BeLLE collaboration, Measurement of the lepton forward-backward asymmetry in $B \rightarrow X_{s} \ell^{+} \ell^{-}$decays with a sum of exclusive modes, Phys. Rev. D 93 (2016) 032008 [Addendum ibid. 93 (2016) 059901] [arXiv: 1402.7134] [INSPIRE].

[98] LHCb collaboration, Rare penguin decays at LHCb, talk given at the 55th Rencontres de Moriond on Electroweak Interactions and Unified Theories, online, 21-27 March 2021, LHCb-TALK-2021-032 (2021).

[99] LHCb collaboration, Measurement of CP-Averaged Observables in the $B^{0} \rightarrow K^{* 0} \mu^{+} \mu^{-}$ Decay, Phys. Rev. Lett. 125 (2020) 011802 [arXiv: 2003.04831] [INSPIRE].

[100] LHCb collaboration, Differential branching fractions and isospin asymmetries of $B \rightarrow K^{(*)} \mu^{+} \mu^{-}$decays, JHEP 06 (2014) 133 [arXiv:1403.8044] [INSPIRE].

[101] LHCb collaboration, Angular analysis and differential branching fraction of the decay $B_{s}^{0} \rightarrow \phi \mu^{+} \mu^{-}, J H E P 09$ (2015) 179 [arXiv:1506.08777] [INSPIRE].

[102] LHCb collaboration, Differential branching fraction and angular analysis of $\Lambda_{b}^{0} \rightarrow \Lambda \mu^{+} \mu^{-}$ decays, JHEP 06 (2015) 115 [Erratum JHEP 09 (2018) 145] [arXiv: 1503. 07138] [INSPIRE].

[103] BELLE collaboration, Search for $B \rightarrow h^{(*)} \nu \bar{\nu}$ with the full Belle $\Upsilon(4 S)$ data sample, Phys. Rev. D 87 (2013) 111103 [arXiv:1303.3719] [INSPIRE].

[104] BABAR collaboration, Search for $B \rightarrow K^{(*)} \nu \bar{\nu}$ and invisible quarkonium decays, Phys. Rev. D 87 (2013) 112005 [arXiv:1303.7465] [INSPIRE].

[105] J. Aebischer, J. Kumar, P. Stangl and D.M. Straub, A Global Likelihood for Precision Constraints and Flavour Anomalies, Eur. Phys. J. C 79 (2019) 509 [arXiv:1810.07698] [INSPIRE].

[106] M. Ciuchini, M. Fedele, E. Franco, A. Paul, L. Silvestrini and M. Valli, Lessons from the $B^{0,+} \rightarrow K^{* 0,+} \mu^{+} \mu^{-}$angular analyses, Phys. Rev. D 103 (2021) 015030 [arXiv: 2011.01212] [INSPIRE].

[107] A. Greljo and D. Marzocca, High-p $p_{T}$ dilepton tails and flavor physics, Eur. Phys. J. C 77 (2017) 548 [arXiv: 1704.09015] [INSPIRE].

[108] J. Fuentes-Martín, A. Greljo, J. Martin Camalich and J.D. Ruiz-Alvarez, Charm physics confronts high- $p_{T}$ lepton tails, JHEP 11 (2020) 080 [arXiv:2003.12421] [INSPIRE]. 
[109] ATLAS collaboration, Prospects for the measurement of $t \bar{t} \gamma$ with the upgraded ATLAS detector at the High-Luminosity LHC, ATL-PHYS-PUB-2018-049 (2018).

[110] CMS collaboration, Anomalous couplings in the $t t+Z$ final state at the $H L-L H C$, CMS-PAS-FTR-18-036 (2018).

[111] CMS collaboration, Projection of measurements of differential ttbar production cross sections in the e/u+jets channels in pp collisions at the HL-LHC, CMS-PAS-FTR-18-015 (2018).

[112] CMS collaboration, Search for new physics in top quark production with additional leptons in proton-proton collisions at $\sqrt{s}=13 \mathrm{TeV}$ using effective field theory, JHEP 03 (2021) 095 [arXiv: 2012.04120] [INSPIRE]. 